\title{
CGRP induction in cystic fibrosis airways alters the submucosal gland progenitor cell niche in mice
}

\author{
Weiliang Xie, ${ }^{1,2}$ John T. Fisher, ${ }^{1}$ Thomas J. Lynch, ${ }^{1}$ Meihui Luo, ${ }^{1}$ Turan I.A. Evans, ${ }^{1}$ \\ Traci L. Neff, ${ }^{3}$ Weihong Zhou, ${ }^{1}$ Yulong Zhang, ${ }^{1}$ Yi Ou, ${ }^{1}$ Nigel W. Bunnett, ${ }^{4}$ Andrew F. Russo, ${ }^{5}$ \\ Michael J. Goodheart, ${ }^{3}$ Kalpaj R. Parekh,6,7 Xiaoming Liu, ${ }^{1,7}$ and John F. Engelhardt1,7

\begin{abstract}
${ }^{1}$ Department of Anatomy and Cell Biology, ${ }^{2}$ Molecular and Cellular Biology Graduate Program, and ${ }^{3}$ Department of Obstetrics and Gynecology, The University of lowa Carver College of Medicine, lowa City, Iowa, USA. ${ }^{4}$ Department of Surgery and Physiology, UCSF, San Francisco, California, USA. ${ }^{5}$ Department of Molecular Physiology and Biophysics, ${ }^{6}$ Department of Cardiothoracic Surgery, and ${ }^{7}$ Center for Gene Therapy of Cystic Fibrosis and Other Genetic Diseases, The University of lowa Carver College of Medicine, Iowa City, Iowa, USA.
\end{abstract}

In cystic fibrosis (CF), a lack of functional CF transmembrane conductance regulator (CFTR) chloride channels causes defective secretion by submucosal glands (SMGs), leading to persistent bacterial infection that damages airways and necessitates tissue repair. SMGs are also important niches for slow-cycling progenitor cells (SCPCs) in the proximal airways, which may be involved in disease-related airway repair. Here, we report that calcitonin gene-related peptide (CGRP) activates CFTR-dependent SMG secretions and that this signaling pathway is hyperactivated in CF human, pig, ferret, and mouse SMGs. Since CGRP-expressing neuroendocrine cells reside in bronchiolar SCPC niches, we hypothesized that the glandular SCPC niche may be dysfunctional in CF. Consistent with this hypothesis, CFTR-deficient mice failed to maintain glandular SCPCs following airway injury. In wild-type mice, CGRP levels increased following airway injury and functioned as an injury-induced mitogen that stimulated SMG progenitor cell proliferation in vivo and altered the proliferative potential of airway progenitors in vitro. Components of the receptor for CGRP (RAMP1 and CLR) were expressed in a very small subset of SCPCs, suggesting that CGRP indirectly stimulates SCPC proliferation in a non-cell-autonomous manner. These findings demonstrate that CGRP-dependent pathways for CFTR activation are abnormally upregulated in CF SMGs and that this sustained mitogenic signal alters properties of the SMG progenitor cell niche in CF airways. This discovery may have important implications for injury/repair mechanisms in the $\mathrm{CF}$ airway.

\section{Introduction}

Stem cell niches in adult organs play important roles in maintaining organ integrity in the setting of normal cellular turnover and injury. These microenvironments are unique and control intrinsic stem cell behavior by providing cell-cell contact with supporting cells, paracrine and endocrine signaling from either local or distant sources, and/or neural input (1). It has become increasingly recognized that dysfunction in the microenvironment of a stem cell niche may contribute to the emergence of disease (1). Conversely, the emergence of disease may impair the function of a stem cell niche and, in turn, contribute to disease progression. The lung is thought to have several stem cell niches for various trophic units, including the tracheobronchial, bronchiolar, and alveolar epithelia (2-4). Submucosal glands (SMGs) in the airway have been proposed to act as a stem cell niche based on the findings that mouse tracheal SMGs retain slow-cycling nucleotide label-retaining cells (LRCs) following injury $(2,5,6)$ and human SMG progenitors are multipotent for differentiation into major surface airway epithelium (SAE) cell types (7).

One major disease that affects airway SMGs is CF. CF results from mutations in the cystic fibrosis transmembrane conductance regulator $(C F T R)$ gene, which expresses a chloride channel at high

Authorship note: John T. Fisher and Thomas J. Lynch contributed equally to this work. Conflict of interest: The authors have declared that no conflict of interest exists. Citation for this article: J Clin Invest. 2011;121(8):3144-3158. doi:10.1172/JCI41857. levels in SMGs (8). Glandular deficiency in CFTR function leads to impaired secretion of fluid into the airways and to a reduction in the secretion of certain factors that are crucial to airway innate immunity $(9,10)$. In humans with $\mathrm{CF}$, glandular secretion is impaired due to the lack of CFTR-mediated chloride transport from the serous cells in the most distal tubules of the glands (9). Although the mouse has relatively few of these glands in its trachea, the tracheal SMGs from Cftr-knockout mice are similar to those in their human CFTR-mutant counterparts with respect to secretory defects $(11,12)$. Hence, it appears that the mouse retains CF-associated glandular defects, making this species attractive for studying glandular cell biology as it relates to CF.

The human CF airway undergoes recurrent injury as a consequence of chronic inflammation caused by persistent bacterial infection, and this leads to elevated levels of repair and cellular proliferation in the airways (13). Superimposed on the enhanced proliferative index of CF airways are disease-dependent changes in SMG cell types that lead to enhanced mucus production (14). These alterations include glandular hypertrophy (expansion of gland mass in existing glands) and metaplasia (replacement of one cell type with another, e.g., replacement of serous cells with mucous cells). Such changes suggest that glandular stem/progenitor cells may have altered biology in CF.

We hypothesized that defective airway SMG secretions in CF could promote compensatory elevations in glandular neuropeptides through sensory neuronal feedback induced by the lack of 

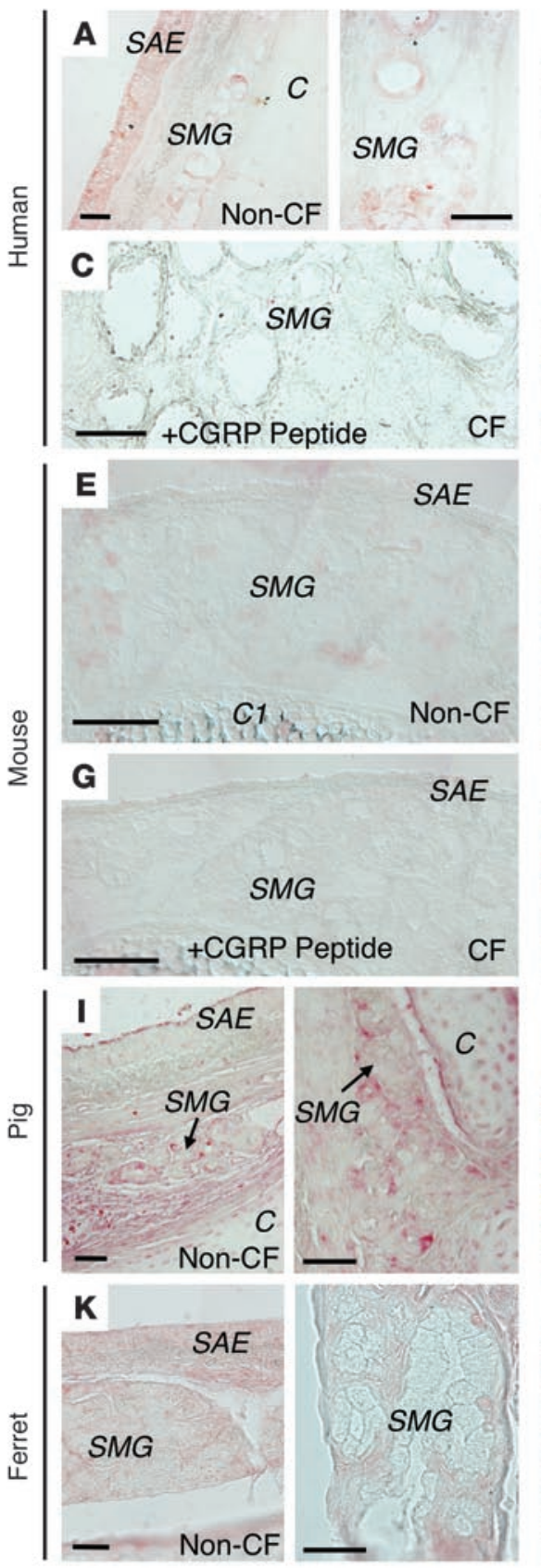
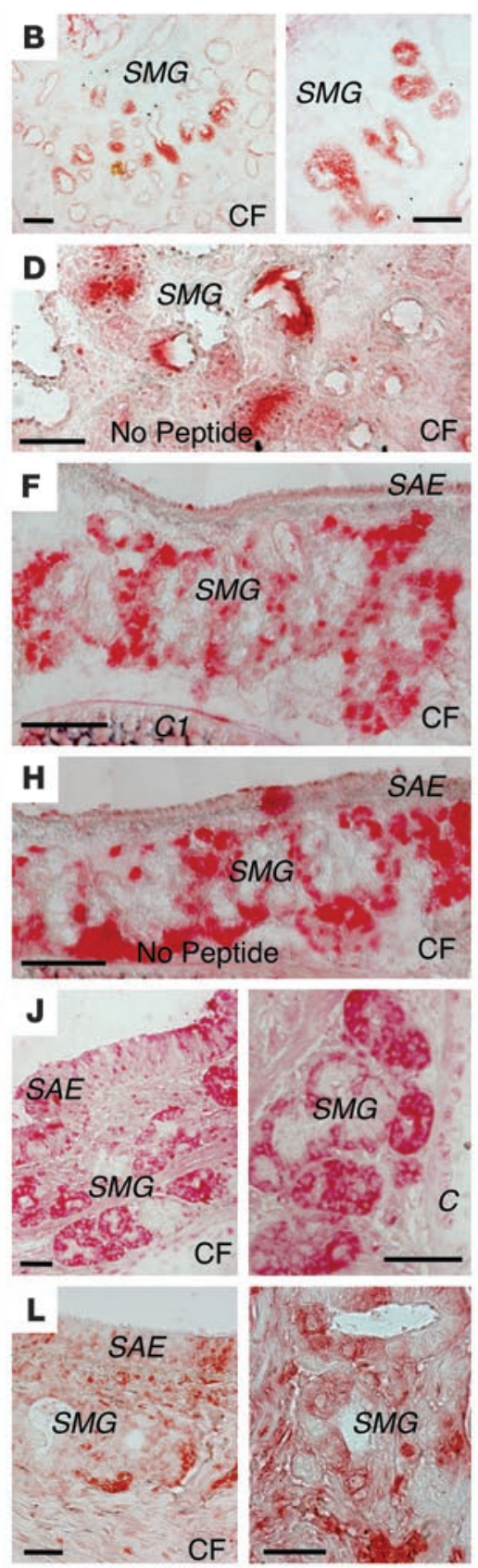
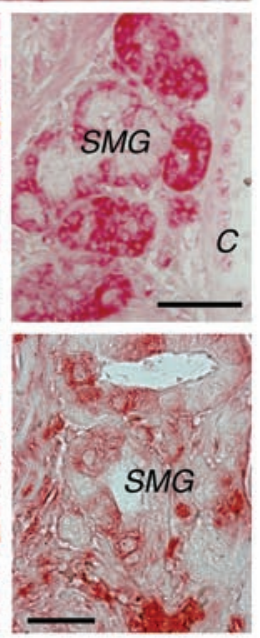

\section{Figure 1}

CGRP expression is elevated in CF human, pig, ferret, and mouse SMGs. Tracheobronchial tissues from non-CF and CF humans (A-D), mouse $(\mathbf{E}-\mathbf{H})$, pig (I and $\mathbf{J})$, and ferret ( $\mathbf{K}$ and $\mathbf{L})$ where histochemically stained for CGRP expression (red staining indicates immunoreactivity; no counterstain was used). As a control for staining specificity, sections from the same block of CF human (D and $\mathbf{C}$ ) and CF mouse ( $\mathbf{G}$ and $\mathbf{H}$ ) tissues were immunostained for CGRP in the absence or presence of $100 \mathrm{ng}$ purified CGRP as indicated. C, cartilaginous ring. Scale bars: $100 \mu \mathrm{m}(\mathrm{E}-\mathrm{H})$, $50 \mu \mathrm{m}$ (A-D and I-L).

induces CFTR-dependent chloride current in airway epithelia and SMG fluid secretion.

Given the association of distal airway slowcycling stem/progenitor cell niches with CGRP-expressing pulmonary neuroendocrine cells (PNECs) $(18,19)$, we hypothesized that elevated expression of CGRP associated with the CF disease state might alter properties of the glandular progenitor cell niche, where slow-cycling LRCs also reside. To test this hypothesis, we evaluated naphthalene-induced tracheal injury responses in Cftr-knockout mice. Results from these studies demonstrated that slow-cycling LRCs shift their anatomical location in the trachea from SMGs (in $\mathrm{Cftr}^{+}$ mice) to the SAE (in $\mathrm{Cftr}^{--}$mice) as a result of the CFTR defect. Colony forming efficiency (CFE) assays evaluating the in vitro proliferative capacity of tracheal progenitors confirmed a shift in anatomical location of highly proliferative progenitors from SMG-containing proximal tracheal segments in $\mathrm{Cftr}^{+/-}$mice to distal tracheal segments lacking SMGs in $\mathrm{Cftr}^{-1}$ mice. Interestingly, naphthalene injury significantly induced CGRP in SMGs of wildtype mice, suggesting that CGRP induction may be involved in activation of the glandular progenitor cell niche following injury. In support of this hypothesis, expression of recombinant CGRP in tracheal SMGs of wild-type mice

CFTR-dependent glandular secretions. Three neuropeptides, including substance P (SP), vasoactive intestinal peptide (VIP), and calcitonin gene-related peptide (CGRP), have been shown to induce SMG secretions $(11,12,15,16)$. To this end, we evaluated expression of these neuropeptides in CF and non-CF SMGs from 4 species, including human, mouse, ferret, and pig. Interestingly, only CGRP was consistently elevated in CF SMGs from all 4 species. Since SP and VIP neuropeptides are known to stimulate CFTR-dependent SMG secretions through the induction of cAMP $(11,12,15)$ and CGRP can also induce cAMP through G protein-coupled receptors (17), we hypothesized that elevated CGRP expression in CF SMGs might be a compensatory mechanism attempting to enhance CFTR-dependent glandular secretions. In support of this hypothesis, we demonstrate that CGRP indeed induced proliferation of glandular progenitors, and treatment of airway epithelial cultures with CGRP peptide stimulated expansion of transient amplifying cells with limited proliferative potential. Based on the cellular localization patterns of the CGRP receptor subunits (receptor activity-modifying protein 1 [RAMP1] and calcitonin receptor-like receptor [CLR]) in SMGs, CGRP appears to indirectly induce proliferation of glandular progenitors though paracrine effector pathways.

To our knowledge, these studies are the first to implicate CGRP as an effector of glandular progenitor cell function in the airway and demonstrate that compensatory dysregulation of this effector pathway in CF leads to adaptive changes in the glandular progenitor cell niche. Such findings may have important implications for airway injury and repair in CF lung disease. 
A Amil
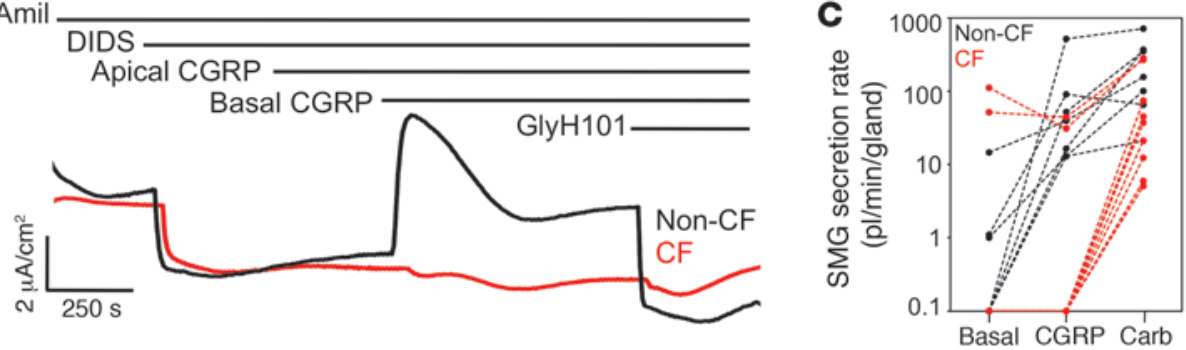

$\mathbf{B}$

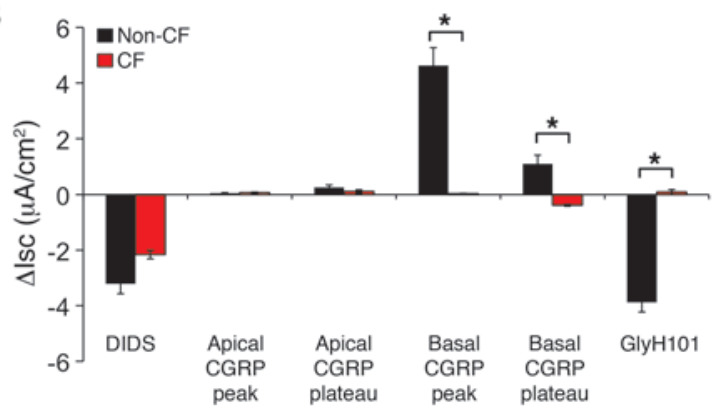

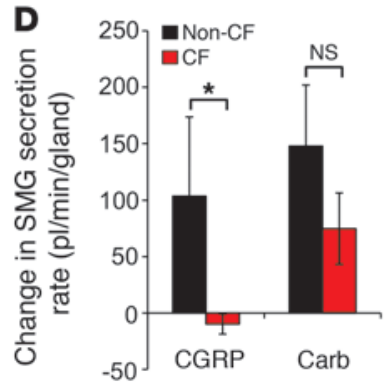

Figure 2

CGRP activates CFTR-mediated chloride transport in airway epithelium and CFTR-dependent tracheal SMG secretion. (A) Example of Isc tracings obtained from polarized CF and non-CF human airway epithelia in response to serial addition of $100 \mu \mathrm{M}$ amiloride (apically), $100 \mu \mathrm{M}$ DIDS (apically), $5 \mathrm{nM}$ CGRP (apically), 5 nM CGRP (basolaterally), and $100 \mu \mathrm{M}$ GlyH101 (apically). (B) Summary of Isc changes for the indicated agonist and antagonist treatments of CF and non-CF human airway epithelia. Results represent the mean \pm SEM for $n=9$ independent measurements for each genotype. (C) Rates of CF $\left(\mathrm{Cftr}^{--}\right)$and non-CF $\left(\mathrm{Cftr}^{+/+}\right)$mouse tracheal SMG fluid secretions at baseline and following serial addition of $10 \mu \mathrm{M}$ CGRP, followed by $10 \mu \mathrm{M}$ carbachol (Carb) to the serosal surface. Fluid secretion rates for each condition over a 10-minute period are plotted (circles) for $n=7$ non-CF glands and $n=9$ CF glands (3-4 independent mice for each genotype). The fluid secretion rates for each gland measured are connected by a dashed line, for visualization of gland-specific changes in secretion to each agonist. (D) Average changes in glandular secretion rates in response to CGRP and carbachol from data in C. Results represent the mean \pm SEM. ${ }^{*} P<0.05$, CF versus non-CF.

\section{Results}

CGRP expression is elevated in CF human, mouse, ferret, and pig SMGs. In CF human, mouse, ferret, and pig, the lack of CFTR expression in SMGs leads to defective glandular secretions (12, 20-22). Several neuropeptides, including VIP, SP, and CGRP, are known to act on SMGs to stimulate secretion $(11,12,15,16)$. We hypothesized that defective glandular secretions in CF could promote compensatory elevations in these neuropeptides through sensory neuronal feedback induced by the lack of CFTR-dependent glandular secretions. To test this possibility, we localized VIP, SP, and CGRP in CF and non-CF tracheobronchial tissues from human, mouse, ferret, and pig. Results from these studies demonstrated that CGRP was consistently upregulated in CF as compared with non-CF SMGs in each of these species (Figure 1). In contrast, no CF-specific differences in VIP or SP staining were noted among the 4 species (data not shown).

CGRP activates CFTR in airway epithelium and stimulates CFTRdependent SMG fluid secretion. We hypothesized that elevated CGRP expression in CF SMGs might be a compensatory mechanism attempting to restore CFTR-dependent secretions. Although other neuropeptides including VIP and SP can directly stimulate CFTR activation in airway epithelia and SMGs, the ability of CGRP to perform similar functions has not been evaluated. To this end, we studied whether CGRP stimulation of human polarized air-

port in airway epithelia and CFTRexpression in CF SMGs is likely due to a compensatory increase in endogenous regulatory signals targeted at enhancing CFTR activation in the setting of CFTR deficiency.

Biologic properties of the SMG slow-cycling progenitor cell niche are altered in CF mouse tracheas. CGRP is a well-known marker of PNECs associated with the bronchiolar stem/progenitor cell niche $(18,19)$. In this context, slow-cycling bronchiolar stem/progenitor cells that retain nucleotide analog label (i.e., LRCs) following airway injury to mice cluster in regions near neuroepithelial bodies (NEBs) that house CGRP-expressing PNECs. PNECs in the bronchiolar airways are thought to provide paracrine factors necessary for maintenance and expansion of distal airway stem/progenitor cells. By contrast, tracheal injury by $\mathrm{SO}_{2}$ or naphthalene leads to the accumulation of slow-cycling LRCs in the most proximal SMGs of mouse trachea found between cartilaginous rings 1 and 2 (C1 and C2) (2, 5). These glandular LRCs are thought to be a stem/progenitor cell population of the proximal airway. Given the association of distal airway stem/progenitor cell niches with CGRP-expressing PNECs (18, 19), we hypothesized that elevated expression of CGRP might alter properties of the glandular progenitor cell niche in the CF airway.

Using nucleotide label retention as a marker of slow-cycling progenitor cells in the trachea, we asked whether CFTR dysfunction in SMGs alters the ability of the tracheal glands to serve as a niche for 

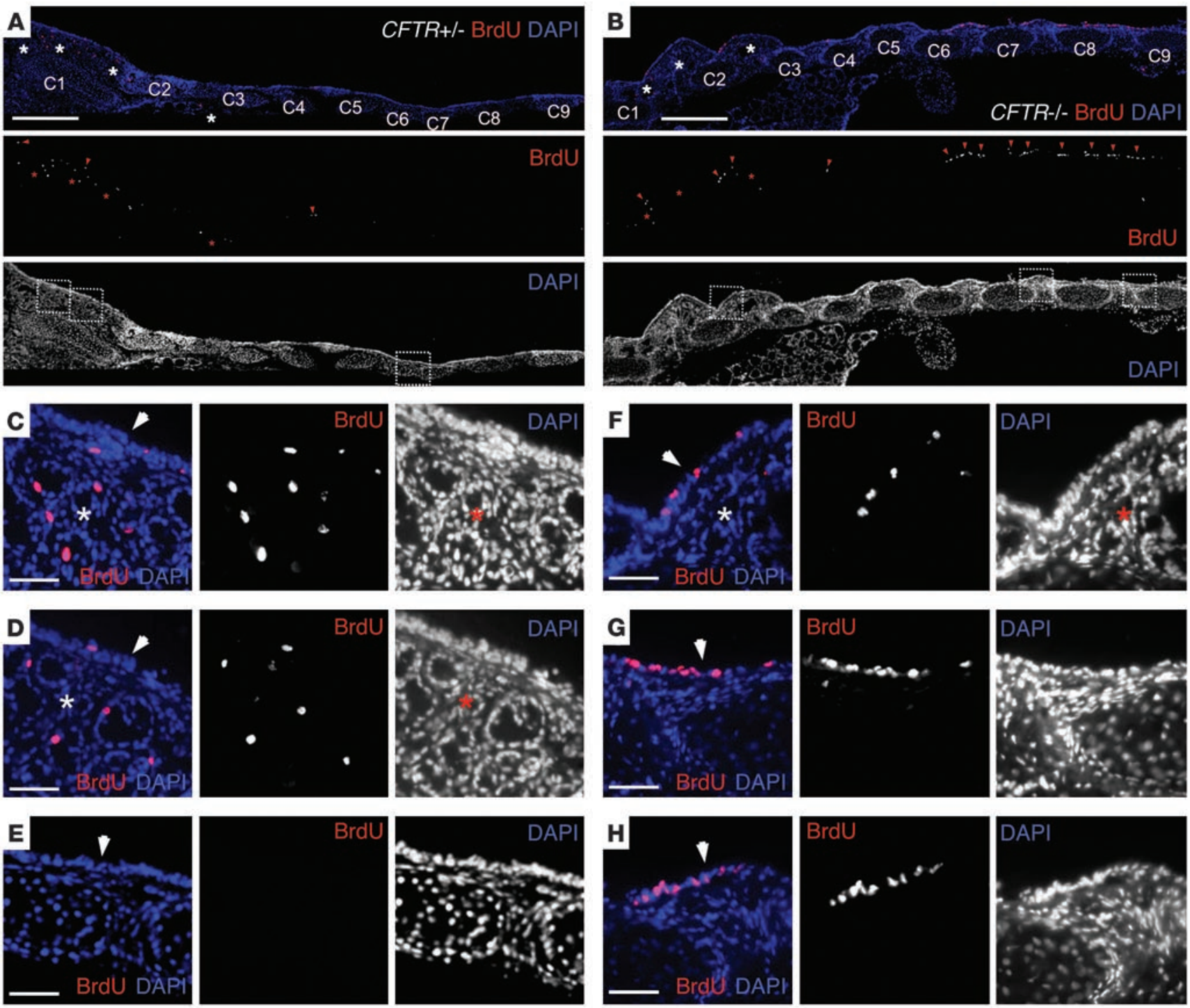

Figure 3

Naphthalene injury promotes LRC relocation from SMGs to the tracheal SAE in Cftr-knockout mice. Naphthalene-injured mice were injected with BrdU 3 times during the first week following injury. At 60 days after injury, tracheal sections were stained for BrdU (red). (A and B) Collages of BrdU-stained tracheas from (A) $\mathrm{Cftr}^{+-}$and (B) $\mathrm{Cftr}^{-1-}$ naphthalene-injured mice. DAPI was used to mark nuclei (blue). The cartilaginous rings of each tracheal section (C1-C9) are labeled in the color images. Below each merged composite are single-channel images for BrdU and DAPI. (C-H) C-E are higher-magnification views of the boxed regions in $\mathbf{A}$. F-H are higher-magnification views of the boxed regions in $\mathbf{B}$. Red and white asterisks mark SMGs; red arrowheads mark BrdU+ cells in the SAE in $\mathbf{A}$ and $\mathbf{B}$; and white arrowheads mark the SAE in $\mathbf{C}-\mathbf{H}$. Scale bars: $200 \mu \mathrm{m}$ (A and B), $25 \mu \mathrm{m}(\mathbf{C}-\mathbf{H})$.

slow-cycling LRCs. To this end, we used naphthalene to injure the airways of homozygous and heterozygous (control) Cftr-knockout mice and compared the localization of LRCs following BrdU labeling at the time of injury (Figures 3 and 4, and Supplemental Figure 1; supplemental material available online with this article; doi:10.1172/JCI41857DS1). Following a 60-day washout period, $96 \%$ of LRCs in $\mathrm{Ctr}^{+/-}$mice resided in the $\mathrm{C} 1-\mathrm{C} 2$ region of the trachea, with the majority (61\%) of LRCs in the trachea residing in SMGs and the remainder localized to the SAE surrounding the ducts of these proximal glands (Figures 3 and 4, and Supplemental Figure 1). In the Cftr ${ }^{+/-}$mice, relatively few LRCs (3.9\%) were found distal to $\mathrm{C} 2$. In the $\mathrm{Cftr}^{-/}$mice, by contrast, the pattern of
LRC localization was significantly $(P<0.0001)$ altered, with only a minority $(7.6 \%)$ of LRCs localized to SMGs in the C1-C2 region, and the vast majority (92.4\%) residing in the SAE and evenly dispersed along the proximal to distal axis of the trachea (C1-C13) (Figures 3 and 4, and Supplemental Figure 1). Despite these significant differences in the localization of tracheal LRCs, the total abundance of LRCs in the two genotypes was similar (109 \pm 19 LRCs in a single longitudinal section of the trachea [C1-C13] of $\mathrm{Cftr}^{-/}$mice, as compared with $101 \pm 14 \mathrm{LRCs}$ in $\mathrm{Cftr}+$ - mice). These findings support the hypothesis that CFTR dysfunction alters the biology of SMGs and prevents this region from serving as a niche for slow-cycling LRCs following tracheal injury. 

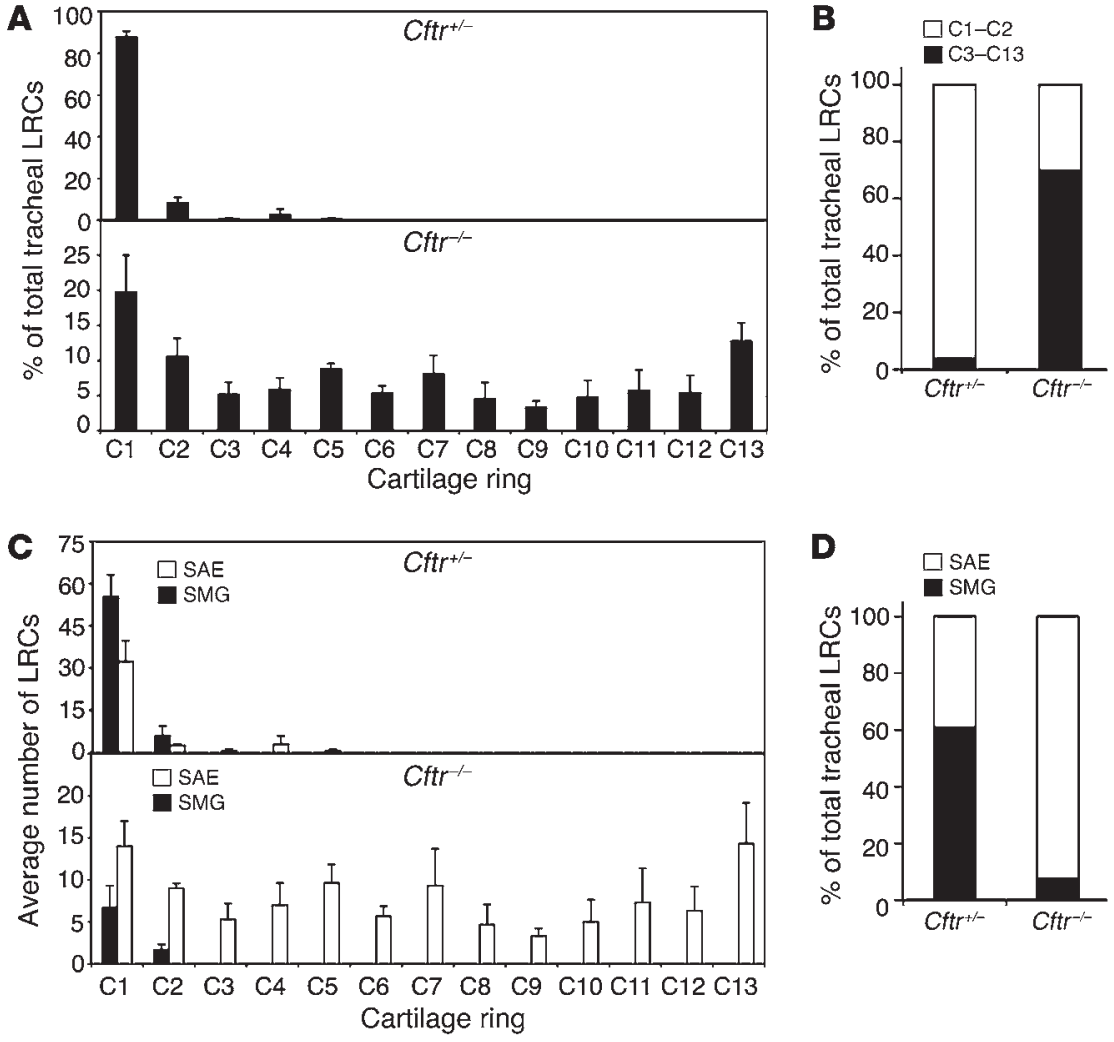

Figure 4

Quantification of LRC localization in the SAE and SMGs following naphthalene injury. (A) MetaMorph software was used to quantify the number of LRCs and their anatomical location throughout longitudinal sections of $\mathrm{Cftr}^{+/-}$and $\mathrm{Cftr}^{-1-}$ tracheas, as indicated. Animals were sacrificed at 60 days after naphthalene injury and BrdU labeling, and for quantification purposes the midpoint between each cartilaginous ring $(\mathrm{C} 1-\mathrm{C} 13)$ was designated as the boundary. Graph depicts the percentage of total LRCs between each cartilaginous ring (includes both SAE- and SMG-derived LRCs) for the indicated genotype. (B) Percentage of all LRCs that were confined to the $\mathrm{C} 1-\mathrm{C} 2$ and $\mathrm{C} 3-\mathrm{C} 13$ regions of the trachea. (C) Average number of LRCs found in the SAE and SMGs between each cartilaginous ring, by genotype. The average total number of LRCs per tracheal section from C1-C13 was $109 \pm 19 \mathrm{for}_{\mathrm{Cft}}^{-1}$ and $101 \pm 14$ for $\mathrm{Cftr}^{+/-}$mice; these numbers did not differ significantly. (D) Percentage of total LRCs localized to the SAE and SMGs, by genotype. All panels depict the mean \pm SEM for $n=3$ animals in each group. Significant differences between the genotypes, as assessed using 2-way ANOVA, were seen for the following 3 comparisons: distribution of total tracheal LRCs relative to the cartilaginous ring $(P<0.0001)$; distribution of glandular LRCs relative to the cartilaginous ring $(P<0.0001)$; and distribution of surface airway epithelial LRCs relative to the cartilaginous ring $(P<0.0001)$.

The distribution of highly proliferative progenitor cells is altered in Cftrknockout mouse tracheas. Label retention is suggestive of a slowcycling phenotype that has been associated with certain stem/ progenitor cell types $(23,24)$. However, rapidly cycling stem cells have also been defined in certain organs that have a high rate of cellular turnover, including the intestine and hair follicle $(25,26)$. Furthermore, terminally differentiated cells can retain label if they persist in an organ for long periods of time. Although the goal of our studies was not to definitively prove that glandular LRCs are indeed multipotent stems cell of the proximal airway, we did seek to evaluate whether the differences in LRC location observed in CF mice reflected alterations in the ability of the glandular niche to retain proliferative progenitors. To this end, we performed CFE assays on SMG-enriched proximal (C1-C6) and
SMG-deficient distal (C7-C13) tracheal segments from $\mathrm{Cftr}^{-/}$and $\mathrm{Cftr}{ }^{+/-}$mice to determine whether LRC localization in the trachea correlated with the potential of progenitor cells to clonally expand in culture. Epithelial cells were isolated from these tracheal segments by pronase digestion and panned to remove fibroblasts prior to assaying of CFE. CFE assays were performed in mixed cultures of ROSA26-LacZ tracheal epithelial cells to allow for the visualization of expanded transgene-negative progenitor clones (Figure 5A). As shown in Figure 5, B-D, both the number of large colony-forming units (>50 cells) and the percent CFE were significantly greater in the gland-rich proximal tracheal segment of $\mathrm{Cftr}^{+/}$mice than in the same region of Cftr ${ }^{-1}$ mice. Additionally, Cftr ${ }^{+/-}$mice demonstrated an approximately 6-fold-higher CFE index in the proximal segment of the trachea $(85 \% \pm 3 \%)$ than in the distal segment $(15 \% \pm 3 \%)$ (Figure 5D), and this correlated with a significantly higher LRC index in the proximal glandular region of the trachea (Figure 4, C and D). Furthermore, the distal segment of the $\mathrm{Cftr}^{-/}$tracheas had a significantly higher (3.5-fold) CFE index than that of the $\mathrm{Cftr}^{+/-}$mice (Figure 5D). Last, the CFE index did not differ significantly between the proximal and distal tracheal segments of $\mathrm{Cftr}^{-/}$ mice (Figure 5D). Despite these genotype-specific differences in the localization of highly proliferative progenitor cells to the proximal and distal segments of the trachea, the total number of large colonies formed per trachea did not differ significantly between genotypes (Figure 5C); on average, $2.5 \times 10^{3}$ cells from $\mathrm{Cftr}^{-1}$ tracheas gave rise to $18 \pm 3$ large colonies, whereas the same number of cells from $\mathrm{Cftr}^{+/}$- tracheas gave rise to $17 \pm 2$ large colonies. Overall, these results are consistent with the finding that LRCs (potential slow-cycling progenitor cells) are differentially dispersed to the proximal glandular region of $\mathrm{Cftr}^{+/-}$tracheas, while being evenly dispersed throughout the trachea in $\mathrm{Ctr}^{-/}$mice (Figure 4C).

To confirm that tracheal slow-cycling LRCs indeed had the capacity to reenter the cell cycle - a requirement for the classification of LRCs as progenitor cells - we examined whether BrdUretaining cells could express cyclin D1 following a second injury. To this end, $C \mathrm{ftr}^{-/}$and $\mathrm{Cftr}{ }^{+/-}$mice were injured with naphthalene and labeled with BrdU and allowed to recover for 90 days to label LRCs. At 90 days the animals were reinjured with naphthalene, and tracheas were harvested at 20 hours after the second injury. These studies revealed a subset of LRCs expressing nuclear cyclin D1 in both the SMGs of Cftr ${ }^{+/-}$mice and the SAE of Cftr-- mice (Figure 6, A-D). These findings support the notion that at least a subset of LRCs from both genotypes has the capacity to reenter the cell cycle and thus can be classified as progenitors. We noted that abundance of tracheal LRCs declined (4-fold) in $\mathrm{Cftr}^{-/}$mice as 
A

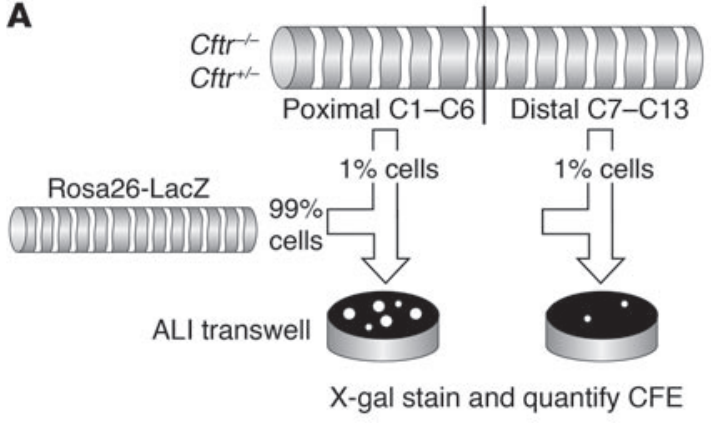

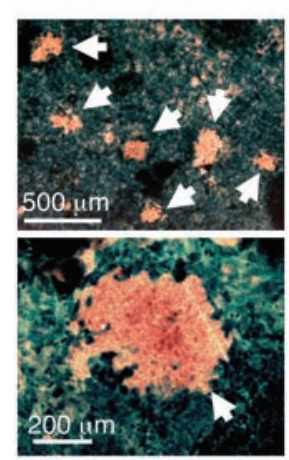

B
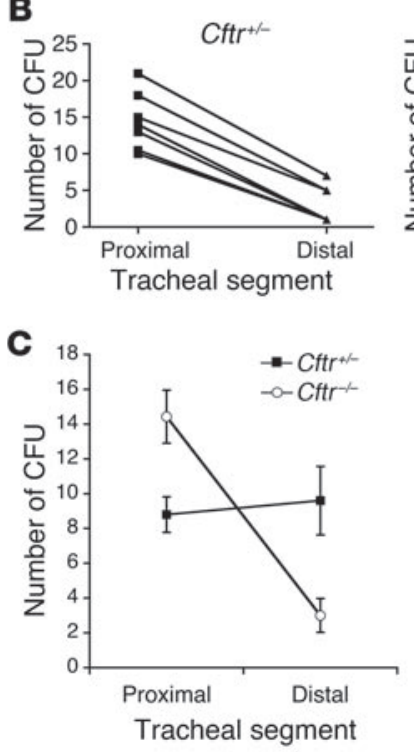
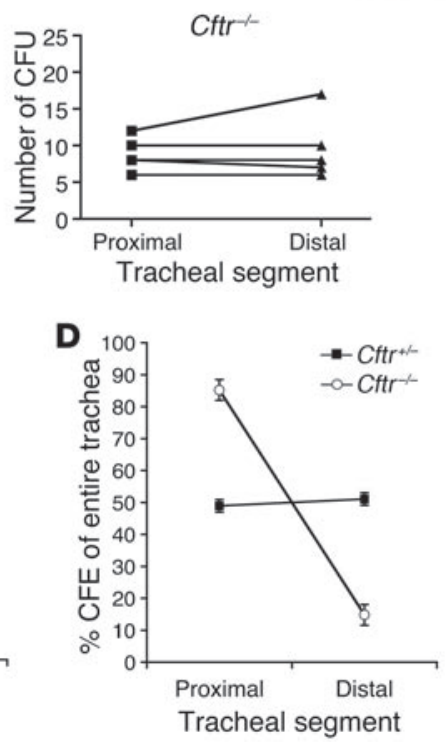

Figure 5

Colony-forming efficiencies of airway progenitors harvested from proximal and distal regions of the trachea. (A) Schematic illustration of the protocol for assessing the CFE index in proximal (C1-C6) and a distal (C7-C13) segment $\mathrm{Cftr}^{-1}$ and $\mathrm{Cftr}^{+-}$mouse tracheas. Photomicrographs on the right demonstrate the formation of transgene-negative colonies (arrows) within a lawn of X-galpositive cells. (B) Total colony-forming units (CFU) in the proximal and distal trachea, as assessed by morphometry (see Methods), for $\mathrm{Cftr}^{+/-}$and $\mathrm{Cttr}^{-1-}$ mice ( $n=5 \mathrm{Cftr}^{-1}$, $n=7 \mathrm{Cftr}^{+/-}$mice in each group). (C) Mean \pm SEM CFU for the data in B. Significant differences, as determined using 1-way ANOVA and Tukey's multiple comparisons test, were seen for the following comparisons: $\mathrm{Cftr}^{+-}$proximal versus $\mathrm{Cftr}^{+-}$distal, $P<0.001 ; \mathrm{Cttr}^{-1}$ proximal versus $\mathrm{Cftr}^{+/}$proximal, $P<0.05 ; \mathrm{Cttr}^{-1}$ distal versus $\mathrm{Cftr}^{+/}$distal, $P<0.05$; $\mathrm{Cftr}^{-1}$ proximal versus $\mathrm{Cftr}^{+/}$- distal, $P<0.05$. No significant difference was seen for $\mathrm{Cftr}^{-1}$ proximal versus $\mathrm{Cftr}^{-1}$ distal, or $\mathrm{Cttr}^{-1-}$ distal versus $\mathrm{Cftr}^{+/-}$proximal. (D) To better evaluate changes in the proximal and distal tracheal regions of a single animal, we normalized the data shown in $\mathbf{C}$ to that for the total tracheal CFU, thus establishing an index of the percent CFE for the proximal and distal regions of the trachea in each animal. Significant differences, as assessed using 1-way ANOVA and Tukey's multiple comparisons test, were seen for all comparisons $(P<0.001)$, except $\mathrm{Cftr}^{-1}$ proximal versus $\mathrm{Cftr}^{-1-}$ distal. compared with $\mathrm{Cft} \mathrm{r}^{+-}$mice following a second injury, despite the fact that the two genotypes retained similar numbers of total LRCs in the trachea prior to a second injury (Supplemental Figure 2). Anatomical quantification of this observation demonstrated that LRCs within non-glandular regions distal to C3 disappeared by 24 hours following a second injury in both genotypes, while LRCs within the gland-rich region of the trachea (C1-C2) only slightly declined (Supplemental Figure 2). The two genotypes demonstrated similar extents of sloughing of the SAE following second injury (Supplemental Figure 3). Since the SAE distal to tracheal C3 in $\mathrm{Cftr}^{--}$mice contained significantly more LRCs than $\mathrm{Cftr}^{+/-}$mice (Figure 4), this accounted for the larger reduction in total tracheal LRCs in $\mathrm{Ctr}^{-/-}$mice following second injury as compared with $\mathrm{Cftr}^{+/-}$mice (Supplemental Figure 2, B and D).

A second approach used to confirm the ability of tracheal LRCs to reenter the cell cycle involved sequential CldU/IdU labeling. In these studies, mice were first labeled with IdU at the time of naphthalene injury, followed by an 80 -day washout period. Mice were then reinjured with naphthalene and pulsed with CldU, followed by a 35-day washout period. Given that $\mathrm{Cftr}{ }^{-1}$ mice demonstrated increased mortality following a second injury, these studies could only be performed in wild-type animals (a significant, $P<0.01$ difference in the survival of $\mathrm{Cftr}^{-/}$and $\mathrm{Ctr}^{+/-}$mice was noted following repeat injury; 8 of $15 \mathrm{Cftr}^{+/-}$animals survived a second naphthalene injury, whereas only 2 of $15 \mathrm{Cftr}^{-1}$ animals survived). Results from this analysis demonstrated that approxi- mately $10 \%$ of IdU-labeled LRCs incorporated and retained CldU label following a second injury (Figure 6, E-I). Given that LRCs presented $0.39 \% \pm 0.03 \%(n=3$ animals $)$ of total glandular cells at 90 days after injury, the abundance of glandular LRCs that reenter the cell cycle following a second injury is a very small percentage of total glandular cells $(0.037 \% \pm 0.005 \%)$. These findings provide strong evidence that a subset of glandular LRCs are slow-cycling progenitor cells capable of reentering the cell cycle following injury.

Glandular CGRP expression is activated following tracheal injury and provides mitogenic signals for progenitor cell expansion by SMGs. CGRPs have been shown to stimulate the proliferation of airway epithelial cells in vitro $(27,28)$. Given this finding, we hypothesized that induction of glandular CGRP might be involved in signaling progenitor cell expansion in SMGs following airway injury and that dysregulation of this mechanism in CF may be responsible for formation of a facultative slow-cycling progenitor cell niche in the SAE. To test this hypothesis, we compared CGRP expression levels in tracheas of $\mathrm{Ctr}^{-/-}$and $\mathrm{Cft}{ }^{+/-}$mice before and after naphthalene injury (Figure 7, A-F). Quantitative morphometric analysis of CGRP immunolocalization to glands within the $\mathrm{C} 1-\mathrm{C} 2$ region demonstrated a significant (9.4-fold; $P<0.005)$ enhancement of expression in the Cftr ${ }^{--}$as compared with the Cftr ${ }^{+/-}$mice (Figure 7G) in the absence of injury, confirming previous observations with histochemical staining for CGRP (Figure 1). Immunoreactivity was also inhibited by preincubation of synthetic CGRP peptide with the primary antibody prior to staining (Figure 7, E and F), demonstrating that 

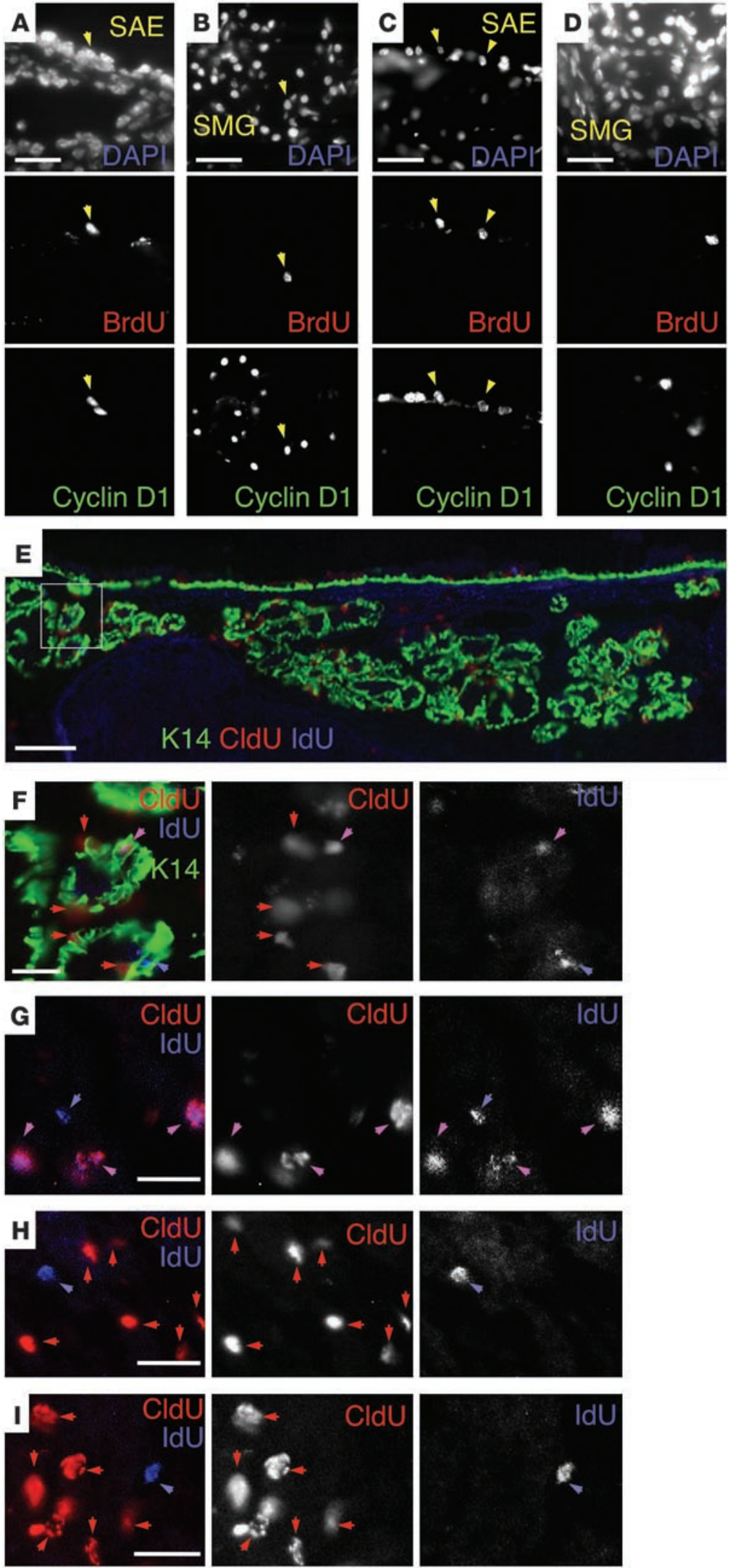

\section{Figure 6}

LRCs in SMGs and the SAE have the capacity to reenter the cell cycle following a second injury. (A-C) Mice were naphthalene injured and labeled with BrdU on days 1, 3 , and 5 after injury. At 90 days after injury, mice were reinjured with naphthalene and euthanized 20 hours later. Tracheal sections were then stained for BrdU (red) and cyclin D1 (green). Photomicrographs depict doublepositive cells (yellow arrowheads) in the SAE and/or SMGs of (A and B) $\mathrm{Cftr}^{+-}$and (C and D) $\mathrm{Cftr}^{-1-}$ mice. (E-I) Wild-type mice were injured with naphthalene and initially labeled with IdU on days 1, 3, and 5 after injury. Animals were subsequently reinjured again at 80 days and labeled with CldU on days 1, 3, and 5 following the second injury. Thirty-five days following the second injury, tracheal sections were costained for CldU, IdU, and/or K14. (E and F) Photomicrographs demonstrating all 3 channels for K14, CldU, and IdU ( $E$ is a collage). $\mathbf{F}$ is an enlargement of the boxed SMG region in E demonstrating one $\mathrm{K}_{14}+\mathrm{CldU}+\mathrm{IdU}+$ cell marked by a purple arrowhead. (G-I) Dual channel images of glandular regions demonstrating single- and double-labeled nuclei marked with colored arrows (IdU+, blue; CldU', red; IdU ${ }^{+} \mathrm{CldU}^{+}$, purple). Photomicrographs show SMG regions with clearly visible singly labeled nuclei ( $\mathbf{H}$ and $\mathbf{I})$, demonstrating the specificity of the staining and clustering of double-positive nuclei (G). Results in all panels are representative of 3 independent mice. Scale bars: $25 \mu \mathrm{m}$ (A-D, F-I), $100 \mu \mathrm{m}$ (E).

the staining was specific. Notably, although naphthalene injury of $\mathrm{Cftr}^{+/-}$mice led to a significant increase (6.4-fold; $P<0.005)$ in CGRP expression in SMGs at 5 days after injury (Figure 7, B, D, and G), this effect was not observed in the SMGs of $\mathrm{Cftr}^{-1}$ mice (Figure 7, A, C, and G). Nevertheless, in both genotypes naphthalene injury increased CGRP immunostaining in the SAE overlying the SMGs (Figure 7, C-E), and this immunostaining was inhibited by the application of synthetic CGRP peptide (Figure 7, E and F). These results were corroborated by ELISA for CGRP using tracheal tissue lysates from the $\mathrm{C} 1-\mathrm{C} 3$ region (Figure $7 \mathrm{H})$; in comparing lysates from uninjured tracheas of $\mathrm{Cftr}^{-/}$and $\mathrm{Cftr}^{+/-}$mice, CGRP levels were 11.7-fold higher in samples generated from the $\mathrm{Cftr}^{-/}$lysates. Our ELISA results also revealed that naphthalene injury induced CGRP levels only in the $\mathrm{Cfr}^{+/-}$mice (14.8-fold increase) (Figure $7 \mathrm{H})$. These findings suggest that elevated production of CGRP in the SMGs of uninjured $\mathrm{Ctr}^{-1-}$ mice may mimic an injured state in wild-type mice. Furthermore, these findings demonstrating upregulation of CGRP in SMGs following airway injury to $\mathrm{Cftr}{ }^{+-}$mice suggest that airway injury likely induces SMG secretion through CFTR. Indeed this was found to be the case (Figure 7I); naphthalene injury increased glandular fluid secretion approximately 6-fold in $\mathrm{Cftr}^{+/-}$mice. Such glandular responses to injury may serve an important role in protecting the injured airway from infection. Given that CGRP is a known mitogen for airway epithelial cells $(27,28)$, we hypothesized that this neuropeptide may function 

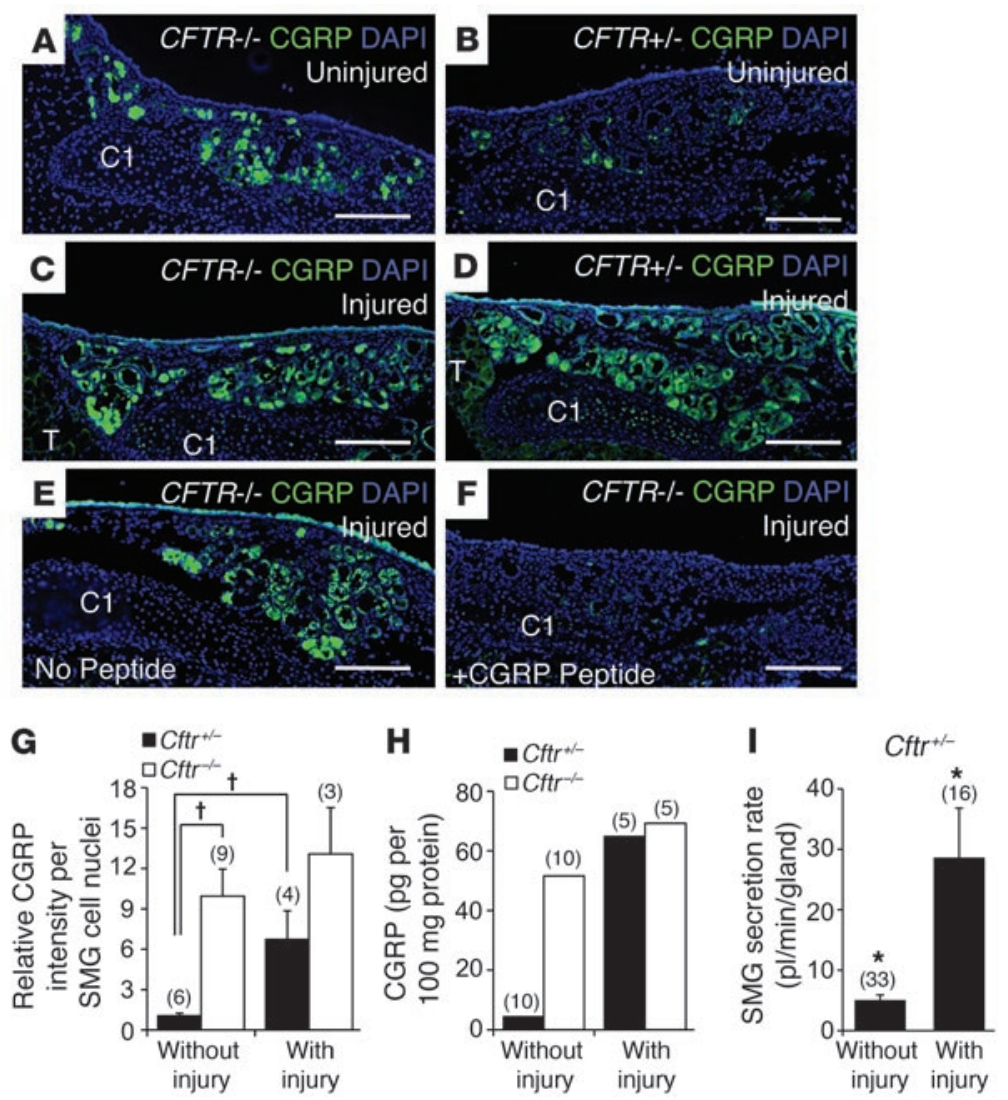

\section{Figure 7}

Glandular CGRP expression is induced following naphthalene injury of $\mathrm{Cftr}^{+-}$but not $\mathrm{Cftr}^{-1}$ mice. (A-D) CGRP expression was evaluated by immunofluorescence in (A and C) $\mathrm{Cttr}^{-1-}$ and (B and D) $\mathrm{Cftr}^{+/-}$mouse tracheas prior to and 5 days following naphthalene injury as indicated. (E and F) CGRP staining of $\mathrm{Cftr}^{-1-}$ tracheal sections from the same animal in the (E) absence or $(\mathbf{F})$ presence of $100 \mathrm{ng}$ purified CGRP. (G) MetaMorph quantification of immunofluorescence staining for experiments represented in A-D. The results represent the relative CGRP staining intensity per glandular nucleus (mean \pm SEM, with the $n$ value shown in parentheses above each bar). Differences between groups were assessed for significance using 1-way ANOVA and the Student's $t$ test, and the marked comparisons were significant $\left({ }^{\dagger} P<0.005\right)$. (H) ELISA-based quantification of CGRP levels for the indicated groups of animals. Because of the low sensitivity of detection, tracheal tissue $(\mathrm{C} 1-\mathrm{C} 3)$ was pooled from 5 animals and assayed in triplicate. The number in parentheses above each bar indicates the total number of animals in each group. The results demonstrate the average amount of CGRP (pg CGRP per $100 \mathrm{mg}$ of protein) in each pooled sample. (I) Glandular secretory rates following naphthalene injury were measured in $\mathrm{Cftr}^{+/-}$mice at 5 days after injury. Results depict the mean \pm SEM, with the value of $n$ glands evaluated for each genotype shown in parentheses above each bar $\left({ }^{*} P<0.005\right)$. Scale bars: $200 \mu \mathrm{m}(\mathbf{A}-\mathbf{F})$. to enhance proliferation of glandular progenitor cells in the injured wild-type trachea and that persistent CGRP signals in $\mathrm{Cftr}^{-1}$ SMGs might alter the ability of the glandular niche to maintain quiescent slow-cycling progenitor cells.

To examine whether enhanced CGRP expression in SMGs induces proliferation of glandular epithelial progenitors, we developed a method to infect tracheal SMGs with recombinant adenovirus. Using this approach we compared BrdU incorporation following infection with a recombinant adenovirus expressing CGRP (Ad. CGRP) or a negative control empty recombinant adenovirus (Ad. Control). Ad.CGRP infection significantly enhanced expression of CGRP in SMGs as determined by immunostaining and ELISA of tracheal lysates, as compared with Ad.Control-infected tracheas (Figure 8, A, B, and G). Overexpression of CGRP led to a significant increase in BrdU incorporation into both SMG and SAE cells of tracheas following a 2-hour pulse. Both CGRP- and non-CGRPexpressing cells were observed to incorporate BrdU label (Figure 8, C-F). BrdU incorporation within the SMGs and SAE was quantified by morphometry (Figure $8 \mathrm{H}$ ) and demonstrated a significant, 16-fold increase in BrdU ${ }^{+}$cells within SMGs and 6.9-fold induction of $\mathrm{BrdU}^{+}$cells within the SAE between $\mathrm{C} 1$ and $\mathrm{C} 2$ following Ad.CGRP infection, as compared with Ad.Control-infected tracheas. These findings demonstrate that CGRP can provide mitotic glandular signals that induce glandular progenitor cells to divide.

CGRP treatment of mixed tracheal airway progenitors expands limited-proliferative-potential transient amplifying cells while reducing the abundance of bighly proliferative progenitor cells. Given that overexpression of CGRP induced SMG progenitor cells to divide in the trachea, we hypothesized that CGRP following tracheal injury might signal slow-cycling progenitors to enter the cell cycle and expand transient amplifying cells (TACs), which in turn regenerate the airway. To begin to approach this hypothesis, we adapted a CFE assay to study the effects of transient CGRP exposure on the proliferative potential of mixed progenitor cell populations isolated from ROSA-26-LacZ and nontransgenic mouse tracheas. In this assay, primary tracheal epithelial cells were seeded on permeabilized supports and exposed to CGRP for the first 2 days in culture. Cultures were allowed to expand for an additional 4 days and then moved to an air-liquid interface (ALI) for an additional 2 weeks. Cultures were then stained, and the abundance and size of LacZ colonies were quantified by morphometry. Results from this experiment demonstrated that CGRP-treated epithelia had a significantly larger number of small colonies (5-10 cells) and fewer medium (11-50 cells) and larger (>50 cells) colonies as compared with untreated controls (Figure 9). Although there was a trend toward more colonies in the CGRP-treated cultures, this trend did not reach statistical significance. These findings suggest that CGRP stimulates expansion of progenitors with limited proliferative potential at the expense of highly proliferative progenitors, a finding consistent with differentiation of early progenitors toward TACs.

$C G R P$ receptor subunits $R A M P 1$ and $C L R$ are primarily expressed in the non-LRC compartment. CGRP signaling requires two integral membrane proteins that form a receptor for CGRP: receptor activity-modifying protein-1 (RAMP1) and calcitonin receptor-like receptor (CLR) $(17,29,30)$. To further evaluate whether CGRP may directly act on slow-cycling progenitor cells to stimulate proliferation, we studied whether RAMP1 and CLR were present on $\mathrm{BrdU}^{+} \mathrm{LRCs}$ in mouse tracheal glands. As shown in Figure 10, both CLR and RAMP1 were expressed in the SAE and SMGs, 

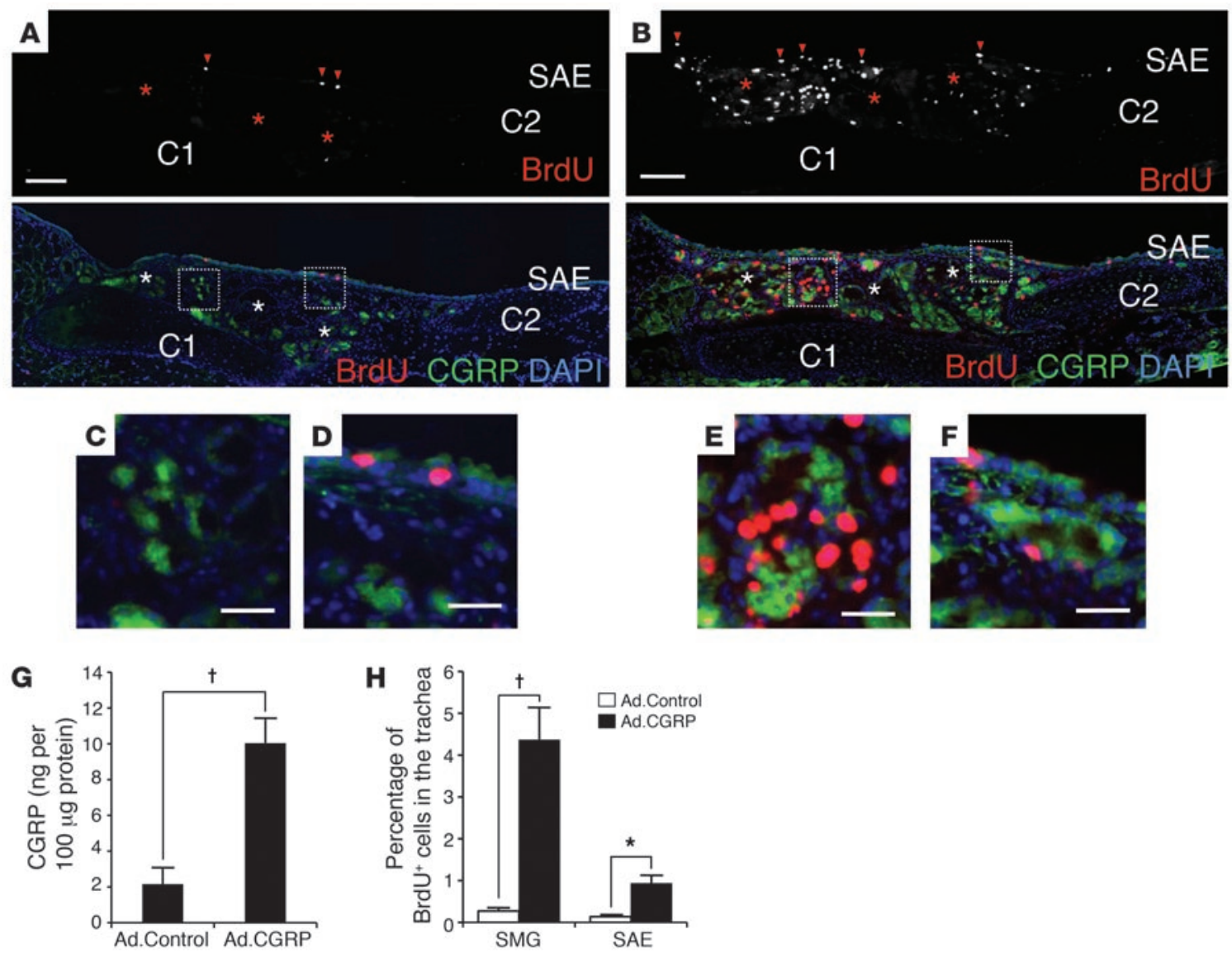

\section{Figure 8}

Overexpression of CGRP in SMGs induces proliferation of glandular progenitor cells. Mouse tracheas were exposed by midline incision, and the exterior of the trachea was infected with recombinant adenovirus $\left(1 \times 10^{12}\right.$ particles) expressing CGRP (Ad.CGRP) or a control virus lacking a transgene (Ad.Control). At 7 days after infection, mice were pulsed with BrdU by i.p. injection for 2 hours, and tracheas were harvested and immunostained for CGRP and BrdU. (A and B) Collages of tracheas from (A) Ad.Control- and (B) Ad.CGRP-infected animals stained for CGRP (green) and BrdU (red). DAPI was used to label nuclei. Top panels represent the BrdU channel alone, and bottom panels represent the merged $\mathrm{BrdU}, \mathrm{CGRP}$, and DAPI channels. Asterisks indicate SMG region; red arrowheads mark BrdU+ cells in the SAE. (C-F) C and D, and E and F are higher-magnification views of the boxed regions in A and B, respectively. (G) Quantification of CGRP expression by ELISA of trachea extracts from animals infected with Ad.CGRP or Ad.Control virus (mean \pm SEM, $n=4$ animals for each group). (H) Quantification of BrdU-labeled nuclei in the SAE and SMGs following infection of the trachea with Ad.CGRP or Ad.Control virus (mean $\pm \mathrm{SEM}, n=5-7$ animals for each group). Differences between marked groups were significantly different: ${ }^{\dagger} P<0.002,{ }^{*} P<0.006$, Student's $t$ test. Scale bars: $100 \mu \mathrm{m}(\mathbf{A}$ and B), $25 \mu \mathrm{m}(\mathbf{C}-\mathbf{F})$.

although CLR was more abundant than RAMP1 in the SMGs. Although these receptors are thought to function at the plasma membrane, the majority of signal appeared intracellular. Such distribution has been reported by others $(31,32)$ and may also be a function of the harsh citrate boiling procedure required for BrdU immunostaining. Morphometric quantification of BrdU, CLR, and RAMP1 demonstrated that $\mathrm{BrdU}^{+} \mathrm{CLR}^{+}$and BrdU $\mathrm{RAMP1}^{+}$ cells were $5.4 \% \pm 0.2 \%$ and $1.4 \% \pm 0.5 \%$ of total glandular $\mathrm{BrdU}^{+}$ LRCs, respectively. Since suitable antibodies for localization of mouse CLR and RAMP1 were both made in rabbits, it was not possible to colocalize both receptors simultaneously to LRCs. Even so, these findings demonstrate that only a small fraction of glandular LRCs expressed either of the CGRP receptor subunits.

\section{Discussion}

CF is characterized by repeated injury/inflammation of the airways of the lung due to chronic bacterial infection. In this setting of continuous insult, the CF lung has increased pressure to regenerate the airway epithelium (13). Currently, it is unclear whether progenitor cell niches in the CF lung are altered as a result of this repetitive injury. SMGs in the proximal airway are one potential progenitor cell niche that may be affected by CF lung disease, since this region is defective in CFTR-mediated fluid secretion and undergoes phenotypic cellular changes with disease progression (i.e., mucous cell hypertrophy). The study described here demonstrates that the biology of the CF mouse tracheal SMG progenitor cell niche is indeed altered by the compensatory induction of CGRP, an activator of CFTR and regulator of CFTR-dependent glandular secretions. Glandular induction of CGRP was also observed in CF human, pig, and ferret tissues. We also observed that airway injury induces glandular expression of CGRP in wildtype mice and that CGRP promotes the division of glandular progenitor cells. Thus, our findings suggest CF SMGs encounter sustained mitogenic pressure - caused by compensatory elevations 
Single cell

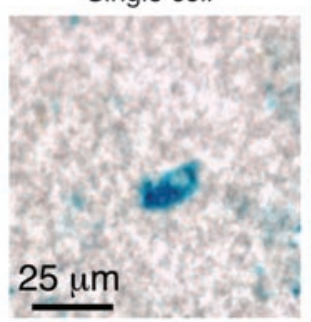

$5-10$ cells

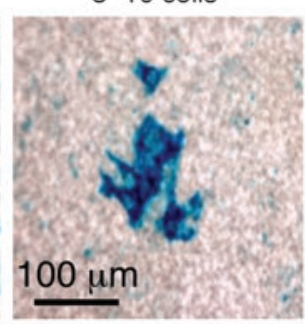

$11-50$ cells

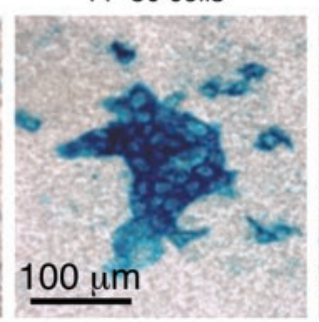

$>50$ cells

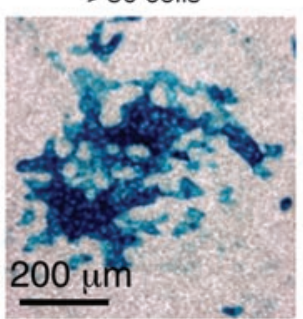

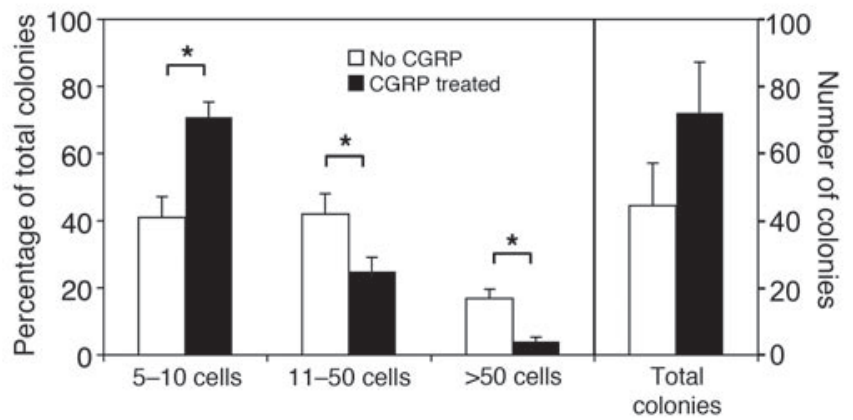

Figure 9

CGRP alters colony-forming efficiencies of tracheal progenitors cells in vitro. In vitro CFE assays were performed using mixed cultures of ROSA-26-LacZ and wild type non-transgenic mouse primary tracheal epithelial cells (1:99 ratio, respectively) in the presence and absence of $100 \mathrm{nM}$ CGRP. Mixed primary tracheal epithelial cells were seeded onto collagen-coated Millicells in the presence or absence of $100 \mathrm{nM}$ CGRP for the first 2 days of growth. Growth medium was then changed (devoid of CGRP), and cultures were allowed to expand for an additional 4 day prior to moving to ALI. After 2 weeks in ALI culture, Millicell filters were stained in X-gal, and collages of the entire filter were quantified for number and size of colonies using MetaMorph software. Examples of colony sizes quantified are given in the top row. Left side of graph shows the distribution of colonies of various sizes for CGRP-treated and untreated cultures. Right side of graph shows the average total number of colonies per Millicell filter for each condition. Data represent the mean \pm SEM for $n=5$ independent cultures for each condition. ${ }^{*} P<0.05$, Student's $t$ test.

in CGRP expression - that leads to adaptive functional changes in slow-cycling progenitor cells of the mouse trachea.

Several findings in this study lend further support to the notion that SMGs in the wild-type mouse trachea serve as a niche for slow-cycling progenitor cells, as first suggested by Borthwick and colleagues $(5,6)$. First, the CFE index for the proximal glandular region of wild-type mouse trachea was significantly higher (6-fold, $P<0.001$ ) than that for the distal portion of the trachea (which lacks SMGs). These findings support the notion that progenitor cells with a high capacity for expansion are enriched in the glandular region of the wild-type trachea. Second, our findings demonstrate that approximately $10 \%$ of glandular LRCs reentered the cell cycle (as indexed by IdU/CldU sequential labeling and cyclin D1 expression) following a second naphthalene injury - consistent with the classification of at least a subpopulation of glandular LRCs as progenitors. Last, sequential IdU/CldU labeling experiments following repeated injury showed that LRCs retained both nucleotide labels after an extended washout period, demonstrating that they remain slow cycling in the setting of repetitive injury. Cumulatively, these in vivo and in vitro analyses support the notion that SMGs harbor slow-cycling progenitor cells for the mouse tracheal airway.

Two pieces of evidence suggest that Cftr-knockout mice have an altered glandular progenitor cell niche. First, only $8 \%$ of tra-

types. Like the LRC index findings, these CFE studies also point toward a change in the distribution, rather than the abundance, of highly proliferative tracheal progenitor cells in the two genotypes. Thus, it appears that the number of tracheal progenitors, as indexed by BrdU label retention and CFE assay, was similar in the two genotypes, despite the difference in localization. These findings support the formation of a facultative progenitor cell niche in the SAE of CF mice.

Several findings support a model whereby compensatory induction of CGRP in CF SMGs leads to alterations to the SMG progenitor cell niche (Figure 11). First, CGRP activates CFTR in airway epithelia and SMGs, and thus elevations in CGRP expression of CF SMGs are consistent with neuronal feedback regulation due to defective CFTR. Second, while the relevant source of CGRP is not yet known, the finding that CGRP acts on the basolateral surface of airway epithelia is consistent with the anatomical sites of autonomic nerve fibers that release neuropeptides such as CGRP. Most nerve fibers that innervate the airways are supplied from the vagus nerve, with cell bodies in the jugular and nodose ganglia and central projections to the medulla $(33,34)$. Some innervation is also provided from dorsal root ganglia. Overstimulation of sensory nerve endings in CF airways and SMGs, due to the lack of functional CFTR, might account for the observed induction of CGRP. Third, expression of recombinant CGRP in wild-type mouse SMGs induced proliferation of glandular progenitors, and following airway injury the tran- 

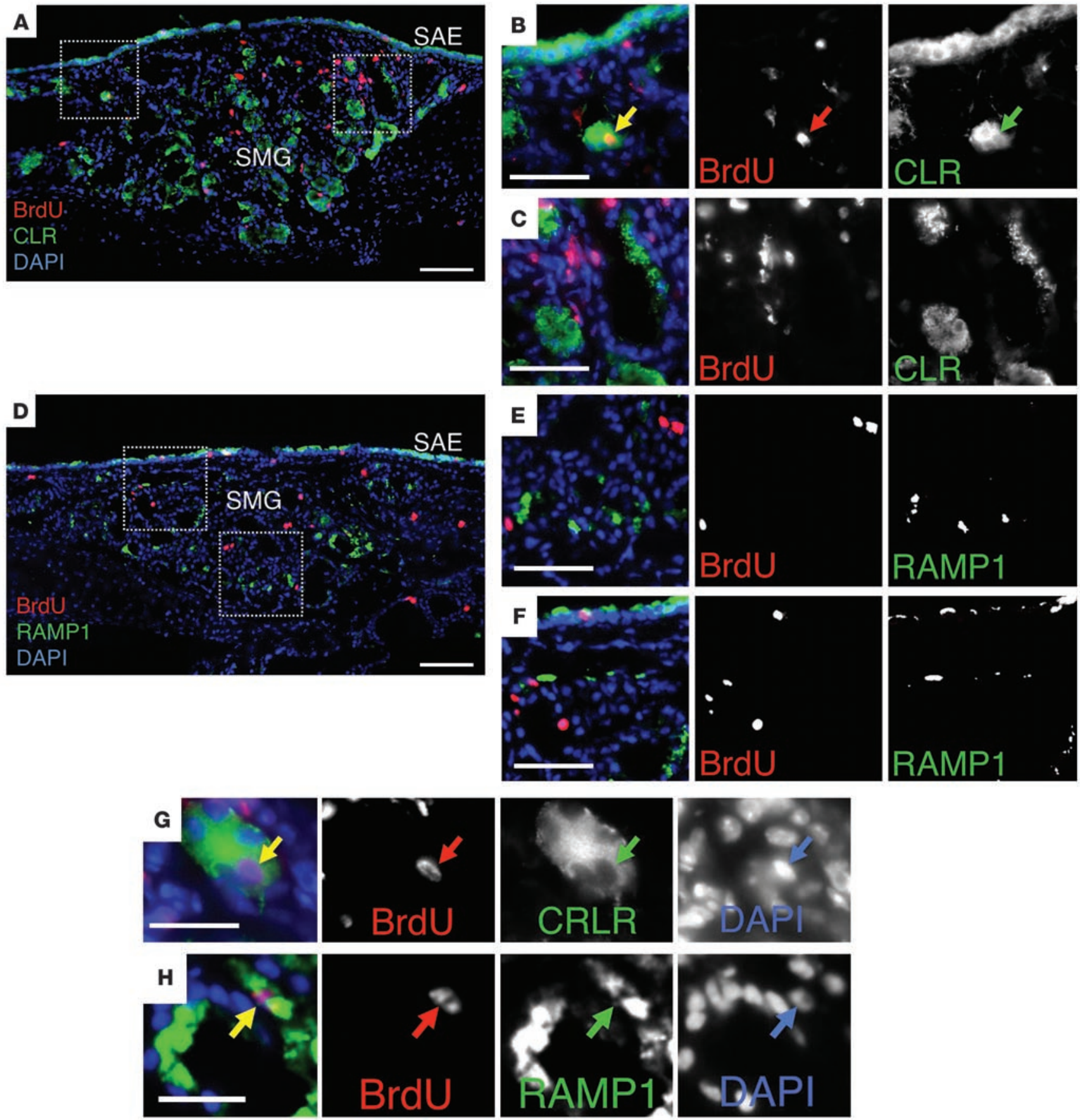

Figure 10

Only a small fraction of glandular LRC progenitors express CGRP receptor components CLR and RAMP1. Wild-type mice were injured with naphthalene and pulse labeled with BrdU on days 1, 3, and 5 after injury. At 60 days following naphthalene injury, tracheas were harvested for co-localization of BrdU with CLR (A-C and $\mathbf{G}$ ) or RAMP1 (D-F and $\mathbf{H})$. B and $\mathbf{C}$ are higher-magnification views of the boxed regions in $\mathbf{A}$. $\mathbf{E}$ and $\mathbf{F}$ are higher-magnification views of the boxed regions in $\mathbf{D}$. Note that $\mathbf{E}$ has been flipped horizontally to avoid label masking of BrdU+ cells. Single-channel images are marked for the various antigen being detected. Arrows mark examples of BrdU $+\mathrm{CLR}^{+}$and BrdU $+R A M P 1+$ cells. Morphometric quantification of 5 independent animals demonstrated that BrdU+CLR+ cells were $5.4 \% \pm 0.2 \%$ of total LRCs and BrdU ${ }^{+} \mathrm{RAMP} 1^{+}$ cells were $1.4 \% \pm 0.5 \%$ of total LRCs; values are mean \pm SEM. Scale bars: $100 \mu \mathrm{m}(\mathbf{A}$ and $\mathbf{D}), 50 \mu \mathrm{m}(\mathbf{B}-\mathbf{F}), 25 \mu \mathrm{m}(\mathbf{G}$ and $\mathbf{H})$.

sient induction of CGRP correlated with cell cycle entry of glandular progenitor cells in wild-type mice. These findings, coupled with the effects of CGRP on CFE indexes, suggest that CGRP acts as a mitogen for slow-cycling progenitors to expand toward tran- sient amplifying progenitors with limited capacity to divide. Thus, we propose a model (Figure 11) whereby elevated and sustained expression of pro-mitotic CGRP in CF SMGs alters the niche milieu to be incompatible with the maintenance of slow-cycling progeni- 

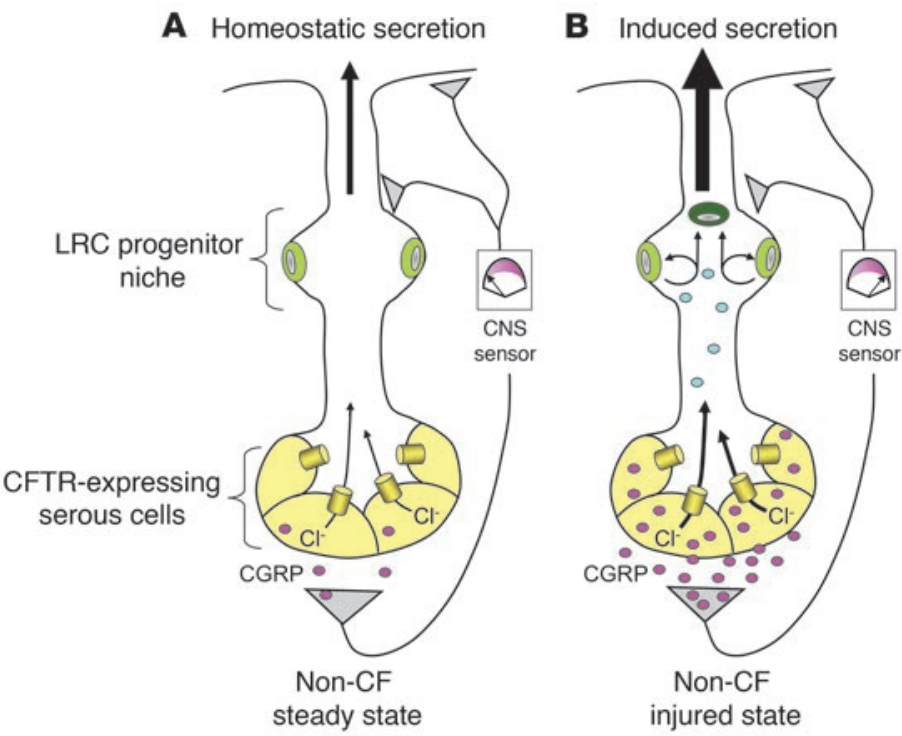

\section{Figure 11}

Working model for CGRP regulation of CFTR-dependent airway gland functions. SMG secretions are controlled by parasympathetic and sympathetic innervation from the CNS. Afferent nerve fibers sense changes in the airway and SMGs, while efferent nerve fibers carry regulatory signals away from the CNS to the airways and SMGs. (A) In the uninjured homeostatic state, nerves produce little CGRP (purple circles), which maintains low-level CFTR-dependent fluid secretion and quiescent LRC progenitors (light green). (B) Following airway injury, sensory neurons send signals to the CNS to enhance CGRP production, which then simulates CFTR-dependent glandular fluid secretion and likely protects the airway from secondary infection. Enhanced CGRP expression also signals glandular LRC progenitors to enter the cell cycle and produce transient amplifying cells (dark green) that affect airway repair. Since CGRP expression is transient, slow-cycling progenitor cells maintain their phenotype within the glandular niche. As shown, the signal to active replication of LRCs is mediated through a second unknown mitogen (small blue circles), since CGRP receptors are very infrequently observed on LRCs. (C) In the CF airway, impaired homeostatic SMG secretions (due to the lack of CFTR) inform the CNS to enhance CGRP production at sites of SMG innervation. Since there is no CFTR to stimulate secretions, feedback signals for production of CGRP remain abnormally high. Prolonged induction of CGRP leads to sustained mitogenic signals in the glandular LRC progenitor niche, and this dysregulation leads to adaptive LRC niche expansion in the SAE.

tor cells. Interestingly, the mitogenic effect of CGRP on guinea pig tracheal epithelial cells has been shown to be lost after 3 days of exposure (28), supporting the notion that sustained CGRP signals in CF SMGs could alter the phenotype of slow-cycling progenitor cells in the glandular niche.

The finding that $C f t r$-knockout mice expand niches for slowcycling progenitors in the SAE is intriguing. A subset of basal cells in the SAE of mouse trachea have also been proposed to be multipotent progenitors and to also have the ability to retain a slow-cycling phenotype $(35,36)$. It remains unclear whether alterations in the anatomical location of slow-cycling progenitor cells in Cftr-knockout mouse tracheas are the result of amplification of resident slow-cycling basal cell progenitors in the SAE and/or relocalization of slow-cycling progenitors out of the SMGs. We favor a model whereby both the SAE and SMGs can function as facultative progenitor cell niches depending on the extent of airway injury. In this setting SMGs may serve as a reservoir for slowcycling progenitors in the face of significant airway injury and denudation. Thus, the most likely explanation of our finding in CF mice is an amplification of the resident SAE basal cell progenitor niche due to persistent mitogenic pressure in the SMG progenitor cell niche caused by maladaptive upregulation of CGRP.

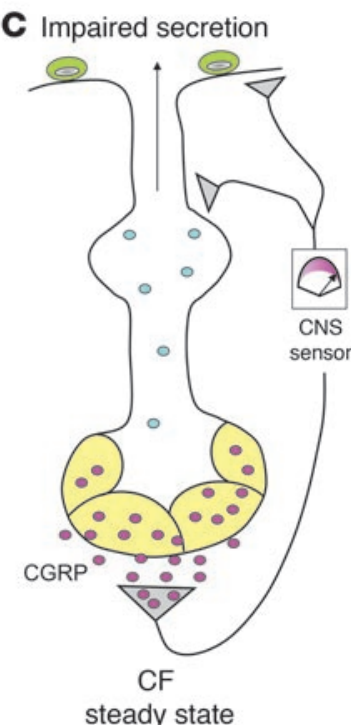

In this setting, CFTR would not directly play a role in regulating progenitor cell phenotype, but rather CGRP pathways that control CFTR-dependent gland secretions become abnormally elevated with the dysregulated consequence of altering the glandular progenitor cell niche.

CGRP is expressed and secreted by PNECs - which are best characterized within the context of $\mathrm{NEBs}$ in the SAE. Interestingly, NEBs have also been associated with the bronchiolar stem cell niche and undergo reactive hyperplasia following naphthalene injury (18), leading to the expansion of CGRP-expressing PNECs. Enhanced production of CGRP in both the distal airway NEBs and SMGs following injury is noteworthy and suggests that CGRP may provide pro-mitotic signals to progenitors in both compartments. The mechanism by which CGRP induces division of slow-cycling SMG progenitor cells remains unclear. However, localization of CLR and RAMP1 coreceptors for CGRP on slowcycling glandular LRCs suggest two potential mechanisms. First, CGRP stimulation may stimulate niche resident non-progenitor cells to secrete factors that act in a paracrine fashion to induce division of slow-cycling glandular progenitor cells. This hypothesis is supported by the finding that $\mathrm{CLR}^{+}$and RAMP1 $1^{+}$LRCs represented only $0.021 \%$ and $0.0055 \%$ of total glandular cells, respectively, a 19- to 71-fold reduction in abundance compared with the frequency of glandular LRCs $(0.39 \%$ of total glandular cells). However, since only approximately $10 \%$ of LRCs reenter the cell cycle following a second injury based on IdU/ CldU sequential labeling, it could alternatively be proposed that low-abundance CLR ${ }^{+}$RAMP1+ $1^{+}$RCs represent a portion of LRCs capable of reentering the cell cycle following direct stimulation by CGRP. However, we believe this second scenario is less likely, since the maximal potential frequency of CLR ${ }^{+}$RAMP1 $1^{+}$LRCs in SMGs $\left(0.0055 \%\right.$ if all RAMP ${ }^{+}$LRCs are also CLR $\left.^{+}\right)$is 6.7 -fold lower than the observed frequency of cycling LRCs (i.e., $\mathrm{IdU}^{+} \mathrm{CldU}^{+}$) in glands following a second injury $(0.037 \% \pm 0.005 \%)$. A definitive conclusion to this question will require the generation of new CGRP receptor antibodies compatible with co-localization of CLR and RAMP1.

In conclusion, our findings provide insights into the functions of CGRP in the injured airway by demonstrating that this neuropeptide controls both CFTR-mediated glandular secretion and proliferation of glandular slow-cycling progenitor cells. Importantly, compensatory hyperactivation of glandular CGRP in CF imposes changes on the glandular progenitor cell niche that may 
have implications for airway injury and repair in the setting of chronic lung infection.

\section{Methods}

Animal procedures. This study was performed according to protocols approved by the Institutional Animal Care and Use Committee of the University of Iowa. Six-week-old $\mathrm{Cftr}^{+/-}$and $\mathrm{Cftr}^{-1-}$ mice - on a CftrmlUnc-Tg (FABPCFTR) 1Jaw/J (stock number 002364, The Jackson Laboratory) background and from the same colony - were used for most studies unless otherwise indicated. All $\mathrm{Ctrr}^{+-}$and $\mathrm{Cftr}^{--}$mice had at least one allele of the FABP-hCFTR transgene to correct for the intestinal obstructive phenotype. For functional studies on tracheal gland secretion, only $\mathrm{Ctr}^{+/+}$ (wild-type) and $\mathrm{Cftr}^{-/}$(knockout) animals were used. Cellular regeneration in the airways was induced by subjection of the mice to naphthalene injury (275 $\mathrm{mg}$ naphthalene/ $\mathrm{kg}$ body weight was injected i.p.), as previously described (2). Slow-cycling LRCs were marked by injection of animals (i.p.) with $80 \mathrm{mg} / \mathrm{kg}$ BrdU 3 times within the first 5 days following naphthalene injury as previously described (2) and outlined in more detail in Supplemental Methods. Tracheal tissue was harvested at 60 days after naphthalene injury for localization of LRCs unless otherwise indicated, embedded into optimal cutting temperature compound (OCT), and cut into longitudinal $10-\mu \mathrm{m}$ sections that spanned cartilaginous rings $\mathrm{C} 1-\mathrm{C} 13$ for immunofluorescence staining. For evaluation of the ability of glandular and surface airway epithelial LRCs to reenter the cell cycle, mice were reinjured with naphthalene at 80-90 days after the first injury and nucleotide labeling. These reinjured animals were sacrificed 20 hours (for cyclin D1 co-localization with BrdU) or 35 days (for CldU/IdU sequential labeling as described below) after undergoing the second injury.

Proximal airway tissues from $\mathrm{CF}$ and non-CF humans, ferrets, and pigs. Human tissues were obtained under a protocol approved by the Institutional Review Board of the University of Iowa. Human bronchial tissues were harvested from $\mathrm{CF}(n=3$ patients, all with $\Delta \mathrm{F} 508 / \Delta \mathrm{F} 508$ genotypes) and nonCF $(n=4$ patients, including 3 lung donors and 1 patient with idiopathic pulmonary fibrosis) individuals at the time of lung transplantation. The $\mathrm{CF}$ and non-CF pig and ferret samples were harvested from the recently reported Cftr-knockout models $(22,37)(n=3$ independent animals for each species and genotype). The pig samples were provided by Michael Welsh and David Stoltz, University of Iowa.

General immunofluorescence and immunohistochemical staining protocols. Immunostaining was performed on $10-\mu \mathrm{m}$ frozen and paraffin-embedded sections using the protocols and antibodies outlined in Supplemental Methods.

Short circuit current measurements of CFTR-mediated chloride transport. Short circuit current (Isc) measurements were performed as previously described (38) using Ussing chambers and polarized human non-CF and CF ( $\Delta$ F508/ $\Delta$ F508) airway epithelia grown at an ALI. Briefly, Millicells were mounted in a P2300/P2302M Ussing chamber system (Physiologic Instruments). Throughout the experiment the chamber was kept at $37^{\circ} \mathrm{C}$, and the chamber solutions were aerated. The basolateral chamber was filled with HEPESbuffered Ringer's solution ( $135 \mathrm{mM} \mathrm{NaCl}, 1.2 \mathrm{mM} \mathrm{CaCl}_{2}, 1.2 \mathrm{mM} \mathrm{MgCl}_{2}$, $2.4 \mathrm{mM} \mathrm{KH}_{2} \mathrm{PO}_{4}, 0.2 \mathrm{mM} \mathrm{K}_{2} \mathrm{HPO}_{4}, 5 \mathrm{mM}$ HEPES, $\mathrm{pH}$ 7.4). The apical chamber received a low-chloride HEPES-buffered Ringer's solution containing a 135 -mM sodium gluconate substitution for $\mathrm{NaCl}$. Isc was recorded using Acquire \& Analyze software (Physiologic Instruments) after clamping the transepithelial voltage to zero. To study CFTR chloride current induced by CGRP, the following antagonists and agonists were sequentially added into the apical or basolateral chamber: (a) apical amiloride $(100 \mu \mathrm{M})$ to block ENaC channels, (b) apical 4,4'-diisothiocyanato-stilbene-2,2'-disulfonic acid (DIDS) $(100 \mu \mathrm{M})$ to block calcium-activated chloride channels, (c) apical CGRP (5 nM), (d) basolateral CGRP (5 nM), and (e) apical $100 \mu \mathrm{M}$ GlyH101 to block CFTR.
Measurements of tracheal SMG secretion rates. Rates of mucus secretion from single mouse trachea SMGs were determined from $\mathrm{CF}\left(\mathrm{Cftr}^{-/}\right)$and non-CF $\left(\mathrm{Cftr}{ }^{++}\right)$mice as previously described (11). Following measurements of baseline secretory rates for 10 minutes, two stimulatory conditions were tested sequentially for 10 minutes with the following solutions: (a) serosal (bath) application of $10 \mu \mathrm{M}$ CGRP and (b) serosal application of $10 \mu \mathrm{M}$ carbachol. Digital images of the spherical mucous bubbles under mineral oil were obtained during baseline and stimulatory conditions, and the spherical volume of each mucus bubble was determined from the diameter of the bubble using a micrometer. The time-dependent changes in mucus bubble volume were then used to calculate secretory rates for individual glands.

Morphometric quantification of immunofluorescence staining. MetaMorph quantification software (Molecular Devices) was used for all morphometric analyses, and the thresholds for all images within a dataset were adjusted using identical parameters. Details on the methods of morphometry are presented in Supplemental Methods.

CFE assays. CFE assays were performed as originally described by Schoch and colleagues (35), with minor modifications that were recently described by our laboratory (2). Briefly, pronase-dissociated cells from each experimental tracheal sample were panned to remove fibroblasts, and the nonadherent cells were quantified prior to mixing together at desire ratio and plating onto collagen-coated Millicells. This mixture was seeded into Millicells and incubated for 6 days in growth medium prior to being moved to an ALI for 2 weeks (2). In studies comparing CFE indexes between CF and non-CF mice, tracheal epithelial cells were harvested from C57BL/6 ROSA26-LacZ, $\mathrm{Ctr}^{-}$-, and $\mathrm{Cft}{ }^{+/}$mice and used to generate various mixed populations for seeding onto Millicells. Tracheas from $\mathrm{Cftr}^{--}$and $\mathrm{Cftr}^{+-}$ mice were divided into two segments (proximal: C1-C6 and distal: C7-C13) prior to the harvesting of tracheal epithelial cells. Tracheal epithelial cells were also harvested from entire tracheas of ROSA26-LacZ mice. $2.5 \times 10^{5}$ cells from pooled ROSA26-LacZ tracheas were allocated for each Millicell and spiked with $2.5 \times 10^{3}$ cells from individual tracheal segments harvested from $\mathrm{Ctr}^{-/}$or $\mathrm{Cftr}+-$ mice (ratio of 100:1). After 2 weeks of ALI culture, filters were stained in X-gal and counterstained in eosin. In this fashion, LacZ transgene-positive cells from ROSA26-LacZ mice were used as the genetic background on which to visualize LacZ-negative clonal outgrowth of $\mathrm{Ctr}^{-/}$or $\mathrm{Cftr}^{+/-}$mouse epithelial progenitors. Filters were excised and photographed under a $5 \times$ objective, and collages were generated using the Photomerge function of Adobe Photoshop. The number of large, LacZnegative colonies (those that were estimated at 50 cells or greater based on the average size of a single cell) was quantified from collages using a MetaMorph Image Analysis system and identical parameters for all groups. Negative control epithelia derived from a pure culture of Rosa26-LacZ tracheal epithelial cells were devoid of LacZ-negative clones.

CFE assays evaluating the effect of CGRP on tracheal progenitor cell expansion used airway epithelial cells harvested from tracheas of Rosa26LacZ and C57BL/ 6 mice. Seeding conditions were identical to that described above, but used a 100:1 ratio of C57BL/6 to ROSA26-LacZ tracheal epithelial cells, respectively. Cultures were exposed to synthetic CGRP peptide (100 nM) (Phoenix Pharmaceuticals Inc., catalog 015-09) or vehicle alone during the first 2 days of culture in growth medium, when non-polarized expansion of epithelial cells occurs. Cultures were then allowed to expand for an additional 4 days in growth medium without CGRP and then moved to an ALI for an additional 2 weeks prior to X-gal staining. The size and abundance of LacZ-positive colonies were then quantified from filter collages using the MetaMorph Image Analysis system. For the CGRP studies, it was possible to quantify a wider range of colony sizes, because the transgenenegative background allows for greater sensitivity in visualization of smaller LacZ-positive colonies. In these studies, colony sizes were grouped into 3 categories (small: 5-10 cells; medium: 11-50 cells; and large: >50 cells). 
Analysis of LRC proliferative index using sequential labeling with IdU and CldU. Mice were injured with naphthalene as described above and labeled on days 1,3 , and 5 with $80 \mathrm{mg} / \mathrm{kg}$ IdU. After an 80-day chase period, mice were reinjured with naphthalene and labeled on days 80,82 , and 84 with $80 \mathrm{mg} / \mathrm{kg}$ CldU. Animals were sacrificed 35 days after the second injury, and tissue was embedded in OCT and cut into $10-\mu \mathrm{m}$ sections. Immunofluorescence staining for IdU and CldU was performed as follows. Samples were fixed, heated in citrate buffer, and blocked as described above for BrdU staining. Slides were then incubated with rat anti-BrdU/CldU antibody (Accurate Chemical and Scientific Corp., OBT0030) at a 1:200 dilution for 2 hours at room temperature and washed twice for 2 minutes in PBS. Slides were incubated in low-salt TBST ( $36 \mathrm{mM}$ Tris, $50 \mathrm{mM} \mathrm{NaCl}, 0.05 \%$ Tween 20 ) at $37^{\circ} \mathrm{C}$ for 30 minutes and then incubated with mouse anti-BrdU/IdU antibody (Roche, 11170376001 ) at a 1:200 dilution and rabbit anti-K14 antibody at a 1:200 dilution (Lab Vision Corp., RB-9020) overnight at $4^{\circ} \mathrm{C}$. K14 labeling was used to assist in localization of SMGs in the absence of DAPI staining to demarcate tubular structures. The following day slides were washed twice for 5 minutes with PBS and then incubated overnight at $4{ }^{\circ} \mathrm{C}$ with a 1:250 dilution of the following secondary antibodies: Texas red-donkey antirat (Jackson ImmunoResearch Laboratories Inc., 712-075-153; minimum cross-reactivity with mouse), AMCA-donkey anti-mouse (Jackson ImmunoResearch Laboratories Inc., 711-095-152; minimum cross reactivity with rat), and FITC-donkey anti-rabbit (Jackson ImmunoResearch Laboratories Inc., 711-095-152). Slides were then washed twice for 5 minutes and coverslipped with VECTASHIELD without DAPI (Vector Laboratories, H-1000). Controls for the specificity of CldU and IdU staining were performed on separate animals labeled separately with each nucleotide and demonstrated no cross-reactivity. The number of $\mathrm{IdU}^{+}$and $\mathrm{IdU}^{+} \mathrm{CldU}^{+}$nuclei within the glandular region (as demarcated by K14 staining) was quantified from at least 25 independent sections from each of 3 animals with a target of quantifying more than $300 \mathrm{IdU}^{+}$cells for CldU staining in each animal.

ELISA for CGRP in tracheal lysates. Tracheal tissue homogenates were generated from the $\mathrm{C} 1-\mathrm{C} 3$ region of the trachea and used for ELISA-based quantification of CGRP expression as described in Supplemental Methods.

Recombinant adenoviral-mediated expression of CGRP in mouse tracheal glands. Recombinant adenovirus was used to assess the functional effects of CGRP expression on the proliferation of glandular progenitor cells. In these studies, the tracheas of 6 - to 8 -week-old wild-type mice were exposed by a midline incision, and $100 \mu \mathrm{l}$ of $1 \times 10^{12}$ particles of recombinant CGRP-expressing adenovirus (Ad.CGRP) (39) or a control virus lacking a transgene (Ad. Control) was injected between the trachea and muscle layer surrounding the trachea. The surgical wound was then closed with sutures. At 7 days after infection, mice were pulsed with $80 \mathrm{mg} / \mathrm{kg} \operatorname{BrdU}$ (i.p.) for 2 hours prior to harvesting of the tracheas for analysis of CGRP expression and BrdU incorporation. Both immunostaining and ELISA were used to quantify CGRP expression and BrdU incorporation in the SAE and SMGs. Using MetaMorph software, the fraction of DAPI-labeled nuclei positive for BrdU in each region was quantified from $n=5-7$ animals in each group.

Statistics. Statistical significance for all comparisons except those for animal survival was assessed using a 2-way ANOVA or a 1-way ANOVA followed by either a Tukey's multiple comparisons test or Student's $t$ test as described in the figure legends. For comparisons of animal survival, Kaplan-Meier survival curves were generated using GraphPad Prism software and compared using the log-rank test; the resulting $P$ values are 2 tailed. A $P$ value less than 0.05 was considered significant.

\section{Acknowledgments}

This work was supported by NIH grants DK047967 and RC1HL099516 (to J.F. Engelhardt), HL091842 (to Michael J. Welsh), DE016511 and NS075599 (to A.F. Russo), and DK39957 (to N.W. Bunnett), and the University of Iowa Center for Gene Therapy (DK54759). We also gratefully acknowledge Christine Blaumueller for editorial assistance and Martin Cassell for helpful scientific discussion.

Received for publication March 25, 2011, and accepted in revised form June 1, 2011.

Address correspondence to: John F. Engelhardt, Department of Anatomy and Cell Biology, University of Iowa, 51 Newton Road, Room 1-111 BSB, Iowa City, Iowa 52242, USA. Phone: 319.335.7744; Fax: 319.335.7198; E-mail: john-engelhardt@uiowa.edu.
1. Scadden DT. The stem-cell niche as an entity of action. Nature. 2006;441(7097):1075-1079.

2. Liu X, Driskell RR, Engelhardt JF. Stem cells in the lung. Methods Enzymol. 2006;419:285-321.

3. Stripp BR. Hierarchical organization of lung progenitor cells: is there an adult lung tissue stem cell? Proc Am Thorac Soc. 2008;5(6):695-698.

4. Randell SH. Airway epithelial stem cells and the pathophysiology of chronic obstructive pulmonary disease. Proc Am Thorac Soc. 2006;3(8):718-725.

5. Borthwick DW, Shahbazian M, Krantz QT, Dorin JR, Randell SH. Evidence for stem-cell niches in the tracheal epithelium. Am J Respir Cell Mol Biol. 2001;24(6):662-670.

6. Engelhardt JF. Stem cell niches in the mouse airway. Am J Respir Cell Mol Biol. 2001;24(6):649-652.

7. Engelhardt JF, Schlossberg H, Yankaskas JR, Dudus L. Progenitor cells of the adult human airway involved in submucosal gland development. Development. 1995;121(7):2031-2046.

8. Engelhardt JF, et al. Submucosal glands are the predominant site of CFTR expression in the human bronchus. Nat Genet. 1992;2(3):240-248.

9. Wine JJ, Joo NS. Submucosal glands and airway defense. Proc Am Thorac Soc. 2004;1(1):47-53.

10. Dajani R, et al. Lysozyme secretion by submucosal glands protects the airway from bacterial infection. Am J Respir Cell Mol Biol. 2005;32(6):548-552.

11. Ianowski JP, Choi JY, Wine JJ, Hanrahan JW. Mucus secretion by single tracheal submucosal glands from normal and cystic fibrosis transmembrane conductance regulator knockout mice. J Physiol. 2007;580(pt 1):301-314.

12. Ianowski JP, Choi JY, Wine JJ, Hanrahan JW. Substance P stimulates CFTR-dependent fluid secretion by mouse tracheal submucosal glands. Pflugers Arch. 2008;457(2):529-537.

13. Leigh MW, Kylander JE, Yankaskas JR, Boucher RC. Cell proliferation in bronchial epithelium and submucosal glands of cystic fibrosis patients. AmJ Respir Cell Mol Biol. 1995;12(6):605-612.

14. Oppenheimer EH, Esterly JR. Pathology of cystic fibrosis review of the literature and comparison with 146 autopsied cases. Perspect Pediatr Pathol. 1975;2:241-278.

15. Choi JY, et al. Substance P stimulates human airway submucosal gland secretion mainly via a CFTR-dependent process. JClin Invest. 2009;119(5):1189-1200.

16. Webber SE, Lim JC, Widdicombe JG. The effects of calcitonin gene-related peptide on submucosal gland secretion and epithelial albumin transport in the ferret trachea in vitro. Br J Pharmacol. 1991; 102(1):79-84.

17. Barwell J, Simms J, Conner A, Hay D, Wheatley M, Poyner D. Ligand binding and activation of the CGRP receptor. In: Hay DL, Dickerson IM, eds. The Calcitonin Gene-Related Peptide Family: Form, Function and Future Perspectives. Doetinchem, The Netherlands: Springer Science; 2010:23-40.

18. Hong KU, Reynolds SD, Giangreco A, Hurley CM,
Stripp BR. Clara cell secretory protein-expressing cells of the airway neuroepithelial body microenvironment include a label-retaining subset and are critical for epithelial renewal after progenitor cell depletion. Am J Respir Cell Mol Biol. 2001;24(6):671-681.

19. Snyder JC, Teisanu RM, Stripp BR. Endogenous lung stem cells and contribution to disease.JPathol. 2009;217(2):254-264.

20. Joo NS, Cho HJ, Khansaheb M, Wine JJ. Hyposecretion of fluid from tracheal submucosal glands of CFTRdeficient pigs. J Clin Invest. 2010;120(9):3161-3166.

21. Lee RJ, Foskett JK. cAMP-activated $\mathrm{Ca}^{2+}$ signaling is required for CFTR-mediated serous cell fluid secretion in porcine and human airways. JClin Invest. 2010; 120(9):3137-3148.

22. Sun X, et al. Disease phenotype of a ferret CFTRknockout model of cystic fibrosis. J Clin Invest. 2010; 120(9):3149-3160.

23. Fuchs E. The tortoise and the hair: slow-cycling cells in the stem cell race. Cell. 2009;137(5):811-819.

24. Leung CT, Coulombe PA, Reed RR. Contribution of olfactory neural stem cells to tissue maintenance and regeneration. Nat Neurosci. 2007;10(6):720-726.

25. Jaks V, et al. Lgr5 marks cycling, yet long-lived, hair follicle stem cells. Nat Genet. 2008;40(11):1291-1299.

26. Barker N, et al. Identification of stem cells in small intestine and colon by marker gene Lgr5. Nature. 2007;449(7165):1003-1007.

27. Kawanami Y, et al. Calcitonin gene-related peptide stimulates proliferation of alveolar epithelial cells. 
Respir Res. 2009;10:8.

28. White SR, Hershenson MB, Sigrist KS, Zimmermann A, Solway J. Proliferation of guinea pig tracheal epithelial cells induced by calcitonin gene-related peptide. Am J Respir Cell Mol Biol. 1993;8(6):592-596.

29. Prado MA, Evans-Bain B, Dickerson IM. Receptor component protein (RCP): a member of a multiprotein complex required for G-protein-coupled signal transduction. Biochem Soc Trans. 2002; 30(4):460-464.

30. Simms J, Hay DL, Wheatley M, Poyner DR. Characterization of the structure of RAMP1 by mutagenesis and molecular modeling. Biophys J. 2006; 91(2):662-669.

31. Cottrell GS, et al. Post-endocytic sorting of calcitonin receptor-like receptor and receptor activity-modifying protein 1.J Biol Chem. 2007;282(16):12260-12271.

32. Zhang Z, Winborn CS, Marquez de Prado B, Russo AF. Sensitization of calcitonin gene-related peptide receptors by receptor activity-modifying protein-1 in the trigeminal ganglion. J Neurosci. 2007; 27(10):2693-2703.

33. Belvisi MG. Overview of the innervation of the lung. Curr Opin Pharmacol. 2002;2(3):211-215.

34. Brouns I, et al. Neurochemical pattern of the complex innervation of neuroepithelial bodies in mouse lungs. Histochem Cell Biol. 2009;131(1):55-74.

35. Schoch KG, Lori A, Burns KA, Eldred T, Olsen JC, Randell SH. A subset of mouse tracheal epithelial basal cells generates large colonies in vitro. $A m J$ Physiol Lung Cell Mol Physiol. 2004;286(4):L631-L642.

36. Ghosh M, et al. Context-dependent differentiation of multipotential keratin 14-expressing tracheal basal cells [published online ahead of print December 3, 2010]. Am J Respir Cell Mol Biol. doi:10.1165/ rcmb.2010-02830C.

37. Rogers CS, et al. Production of CFTR-null and CFTR$\Delta$ F508 heterozygous pigs by adeno-associated virusmediated gene targeting and somatic cell nuclear transfer. J Clin Invest. 2008;118(4):1571-1577.

38. Liu X, Luo M, Zhang L, Ding W, Yan Z, Engelhardt JF. Bioelectric properties of chloride channels in human, pig, ferret, and mouse airway epithelia. Am J Respir Cell Mol Biol. 2007;36(3):313-323.

39. Toyoda K, Faraci FM, Russo AF, Davidson BL, Heistad DD. Gene transfer of calcitonin gene-related peptide to cerebral arteries. Am J Physiol Heart Circ Physiol. 2000;278(2):H586-H594. 